JOURNAL OF THE

AMERICAN MATHEMATICAL SOCIETY

Volume 10, Number 2, April 1997, Pages 259-281

S 0894-0347(97)00228-2

\title{
DELIGNE PERIODS OF MIXED MOTIVES, $K$-THEORY AND THE ENTROPY OF CERTAIN $\mathbb{Z}^{n}$-ACTIONS
}

\author{
CHRISTOPHER DENINGER
}

\section{INTRODUCTION}

For a coherent sheaf $\mathcal{M}$ on the split $n$-torus $\mathbb{G}_{m, A}^{n}=\operatorname{spec} A\left[\mathbb{Z}^{n}\right]$ over a commutative ring $A$ there is a natural $\mathbb{Z}^{n}$-action on its group of global sections $\Gamma(\mathcal{M})=$ $\Gamma\left(\mathbb{G}_{m, A}^{n}, \mathcal{M}\right)$. If we give $\Gamma(\mathcal{M})$ the discrete topology, we obtain a $\mathbb{Z}^{n}$-action on the compact abelian Pontrjagin dual $\Gamma(\mathcal{M})^{*}$. The dynamical properties of this action for $A=\mathbb{Z}$ have been investigated for some time, especially by K. Schmidt. His book [Sch] gives a comprehensive account of the theory developed so far. In particular, one may ask for the entropy $0 \leq h(\mathcal{M}) \leq \infty$ of the $\mathbb{Z}^{n}$-action on $\Gamma(\mathcal{M})^{*}$, which measures to what extent repeated application of the action "scatters around points". Note that for any action of $\mathbb{Z}^{n}$ on a compact abelian group, topological entropy and metric entropy with respect to Haar measure coincide. The results of Lind, Schmidt and Ward [LSW] (see also [Sch], Chap. V) can be formulated as follows $(A=\mathbb{Z})$ : The entropy of $\mathcal{M}$ is finite if and only if $\mathcal{M}$ belongs to the category $\mathcal{T}$ of coherent torsion sheaves on $\mathbb{G}_{m, \mathbb{Z}}^{n}$. By Yuzvinskii's addition formula [Sch], Th. 14.1, entropy defines a homomorphism from the Grothendieck group of $\mathcal{T}$ to the reals:

$$
h: K_{0}(\mathcal{T}) \longrightarrow \mathbb{R},
$$

so that it suffices to calculate $h\left(\mathcal{O}_{Z}\right)$ for irreducible closed subschemes $Z \subset \mathbb{G}_{m, \mathbb{Z}}^{n}$ with $Z \neq \mathbb{G}_{m, \mathbb{Z}}^{n}$. If $Z$ is not defined by a Laurent polynomial, then $h\left(\mathcal{O}_{Z}\right)=0$. On the other hand, for any

$$
0 \neq P \in \Gamma\left(\mathbb{G}_{m, \mathbb{Z}}^{n}, \mathcal{O}\right)=\mathbb{Z}\left[\mathbb{Z}^{n}\right]=\mathbb{Z}\left[t_{1}^{ \pm 1}, \ldots, t_{n}^{ \pm 1}\right]
$$

setting $Z=\operatorname{spec}\left(\mathbb{Z}\left[\mathbb{Z}^{n}\right] /(P)\right)$, they show that

$$
h\left(\mathcal{O}_{Z}\right)=m(P):=\frac{1}{(2 \pi i)^{n}} \int_{T^{n}} \log \left|P\left(z_{1}, \ldots, z_{n}\right)\right| \frac{d z_{1}}{z_{1}} \cdots \frac{d z_{n}}{z_{n}},
$$

where $T^{n}=S^{1} \times \cdots \times S^{1}$ is the real $n$-torus. The quantity $M(P)=\exp m(P)$ is the so called Mahler measure of the polynomial $P$. In transcendence theory $M(P)$ can be used to define a local height at infinity for polynomials in several variables; cf. [Ph]. The numbers $m(P)$ are also related to Lehmer's conjecture, which could be settled if one could prove that the set of values $m(P)$ for $0 \neq P \in \mathbb{Z}\left[\mathbb{Z}^{n}\right], n \geq 1$, is closed in the topology of $\mathbb{R}$; cf. [Bo1].

Received by the editors May 7, 1996.

1991 Mathematics Subject Classification. Primary 11G40, 19E08; Secondary 28D20.

Key words and phrases. Dynamical systems of algebraic origin, extensions of mixed motives, Beilinson conjectures, algebraic $K$-theory, entropy of $\mathbb{Z}^{n}$-actions.

(C) 1997 American Mathematical Society 
The present paper is based on the observation that in the definition of $m(P)$ we integrate with respect to a differential form which appears in connection with the $(n+1)$-fold cup product $\log |P| \cup \log \left|z_{1}\right| \cup \cdots \cup \log \left|z_{n}\right|$ in the real Deligne cohomology of $\mathbb{G}_{m, \mathbb{R}}^{n} \backslash Z_{\mathbb{R}}$. If $P$ does not vanish on $T^{n}$ or equivalently if the $\mathbb{Z}^{n}$-action on $\Gamma\left(\mathcal{O}_{Z}\right)^{*}$ is expansive, $m(P)$ can actually be obtained by evaluating this cup product on the homology class defined by $\left[T^{n}\right]$. Since $\log |P| \cup \log \left|z_{1}\right| \cup \cdots \cup \log \left|z_{n}\right|$ is the image under the regulator map of the symbol $\left\{P, t_{1}, \ldots, t_{n}\right\}$ in the algebraic $K$-theory of $\mathbb{G}_{m, \mathbb{Z}}^{n} \backslash Z$, there is a fundamental relation between $m(P)$ and higher $K$-theory. We also describe explicitly the mixed motive which corresponds to $\left\{P, t_{1}, \ldots, t_{n}\right\}$ and prove directly that $m(P)$ is one of its Deligne periods.

If $P$ vanishes on $T^{n}$, it becomes more difficult to interpret $m(P)$ in homological terms. Under certain assumptions on $P$ however we can express $m(P)$ as the difference of two symbols evaluated against topological cycles or equivalently as a difference of two Deligne periods of certain mixed motives. In one example we indicate briefly how a formula such as ([Sch], VI, 19.10)

$$
m\left(\left(t_{1}+t_{2}\right)^{2}+3\right)=\frac{2}{3} \log 3+\frac{\sqrt{3}}{\pi} L\left(\chi_{3}, 2\right),
$$

where $\chi_{3}:(\mathbb{Z} / 3)^{*} \rightarrow \mu_{2}$ is the nontrivial character, can be deduced mod $\mathbb{Q}^{*}$ from known cases of the Beilinson conjectures. A number of formulas like (2) have been discovered by Smyth [Sm], Boyd [Bo1] and Ray [R] among others. Our approach in conjunction with the Beilinson conjectures clarifies the relation between $m(P)$ and special values of $L$-series that they observed in the examples. A further example concerns $m(P)$ for the Laurent polynomial

$$
P\left(t_{1}, t_{2}\right)=t_{1}^{-1}+t_{2}^{-1}+1+t_{1}+t_{2},
$$

suggested to us by D.W. Boyd. It turns out that $m(P)$ is essentially given by a value of an Eisenstein Kronecker series. We also show that if the rank of a certain $K$-group is as expected, the following formula holds:

$$
m\left(t_{1}^{-1}+t_{2}^{-1}+1+t_{1}+t_{2}\right)=c(2 \pi)^{-2} L(E, 2)
$$

for some $c \in \mathbb{Q}^{*}$. Here $L(E, s)$ is the $L$-function of the elliptic curve $E / \mathbb{Q}$ obtained by taking the projective closure of $P=0$ and specifying a suitable origin. Numerical evidence obtained subsequently by D.W. Boyd suggests that in fact $c=15$. Prompted by this example he in fact discovered many more cases numerically where Mahler's measures are apparently related to $L$-values of elliptic curves [Bo2]. I was also informed that further relations of Mahler measures with Eisenstein Kronecker series have since been obtained by F.R. Villegas.

Some results of this paper were announced in [D3], where a short introduction to mixed motives can also be found. A much more thorough treatment of motives is given in [JKS]. For the Beilinson conjectures and related notions like Deligne cohomology we recommend the book [RSS]. Some observations of the present paper may be of interest to researchers in fields of mathematics other than arithmetic geometry, e.g. in dynamical systems. For this reason I have occasionally recalled standard material in the first two sections.

It is a pleasure for me to thank Y. Ihara for the invitation to Kyoto and the RIMS for support. I would also like to thank D.W. Boyd for interesting correspondence and for bringing the above-mentioned polynomial to my attention. 


\section{Contents.}

1. Symbols and Mahler measure in the expansive case

2. Mahler measure and periods of mixed motives in the expansive case

3. Symbols and the difference of two Mahler measures Appendix: Jensen's formula and the tame symbol

4. Periods of mixed motives and the difference of two Mahler measures

\section{Symbols and Mahler measure in the expansive CASE}

In this section we interpret $m(P)$ if $P$ is nonzero on $T^{n}$ as a Deligne cohomology class evaluated against the homology class defined by $T^{n}$.

For a smooth variety $X$ over $\mathbb{C}$ the Deligne cohomology groups with real coefficients $H_{\mathcal{D}}^{i}(X / \mathbb{C}, \mathbb{R}(j))=H_{\mathcal{D}}^{i}(X, \mathbb{R}(j))$ are defined as certain hypercohomology groups [B1], Ch. 1, [Schn]. The groups $H_{\mathcal{D}}^{i}(X, \mathbb{R}(i))$ that we are interested in have the following description in terms of $C^{\infty}$-forms:

$$
\begin{aligned}
& H_{\mathcal{D}}^{i}(X, \mathbb{R}(i)) \\
& \quad=\left\{\varphi \in \mathcal{A}^{i-1}(X, \mathbb{R}(i-1)) \mid d \varphi=\pi_{i-1}(\omega), \omega \in F^{i}(X)\right\} / d \mathcal{A}^{i-2}(X, \mathbb{R}(i-1)) .
\end{aligned}
$$

On the right-hand side we have written $X$ for $X(\mathbb{C})$ with its structure as an analytic manifold. By $\mathcal{A}^{i}(X, \mathbb{R}(j))$ we denote the space of $\mathbb{R}(j)=(2 \pi \sqrt{-1})^{j} \mathbb{R} \subset \mathbb{C}$-valued smooth $i$-forms on $X$, and $F^{i}(X)$ is the space of holomorphic $i$-forms on $X$ with at most logarithmic singularities at infinity. Finally $\pi_{n}: \mathbb{C} \rightarrow \mathbb{R}(n)$ is the canonical projection $\pi_{n}(z)=\frac{1}{2}\left(z+(-1)^{n} \bar{z}\right)$. The cup product

$$
\cup: H_{\mathcal{D}}^{i}(X, \mathbb{R}(i)) \times H_{\mathcal{D}}^{j}(X, \mathbb{R}(j)) \longrightarrow H_{\mathcal{D}}^{i+j}(X, \mathbb{R}(i+j))
$$

on Deligne cohomology has the following explicit description: For $\alpha=i, j$ consider forms:

$$
\varphi_{\alpha} \in \mathcal{A}^{\alpha-1}(X, \mathbb{R}(\alpha-1)) \quad \text { with } \quad d \varphi_{\alpha}=\pi_{\alpha-1}\left(\omega_{\alpha}\right), \quad \text { where } \omega_{\alpha} \in F^{\alpha}(X),
$$

and the corresponding cohomology class $\left[\varphi_{\alpha}\right]$ in $H_{\mathcal{D}}^{\alpha}(X, \mathbb{R}(\alpha))$. Setting

$$
\varphi_{i} \cup \varphi_{j}=\varphi_{i} \wedge \pi_{j} \omega_{j}+(-1)^{i} \pi_{i} \omega_{i} \wedge \varphi_{j} \quad \text { in } \mathcal{A}^{i+j-1}(X, \mathbb{R}(i+j-1))
$$

one checks that

$$
d\left(\varphi_{i} \cup \varphi_{j}\right)=\pi_{i+j-1}\left(\omega_{i} \wedge \omega_{j}\right)
$$

The cup product of the classes $\left[\varphi_{i}\right]$ and $\left[\varphi_{j}\right]$ is given by

$$
\left[\varphi_{i}\right] \cup\left[\varphi_{j}\right]=\left[\varphi_{i} \cup \varphi_{j}\right] \in H_{\mathcal{D}}^{i+j}(X, \mathbb{R}(i+j)) .
$$

On cohomology $\cup$ is associative and graded commutative, whereas on the level of forms $\cup$ is not associative. In the following case however, which is essential for us, there is a canonical form representing iterated cup product.

Lemma 1.1 ([B2], 2.2, [D1], (7.2)). For $n \geq 0$ consider elements

$$
\varepsilon_{0}, \ldots, \varepsilon_{n} \in H_{\mathcal{D}}^{1}(X, \mathbb{R}(1))=\left\{\varepsilon \in \mathcal{A}^{0}(X, \mathbb{R}) \mid d \varepsilon=\pi_{0}(\omega), \omega \in F^{1}(X)\right\} .
$$

Define a smooth $\mathbb{R}(n)$-valued $n$-form on $X$ by

$$
\begin{aligned}
C_{n+1} & =C_{n+1}\left(\varepsilon_{0}, \ldots, \varepsilon_{n}\right) \\
& =2^{n} \sum_{i=0}^{n}(-1)^{i} \frac{1}{(n+1) !} \sum_{\sigma \in \mathfrak{S}_{n+1}} \operatorname{sgn} \sigma \varepsilon_{\sigma_{0}} \bar{\partial} \varepsilon_{\sigma_{1}} \cdots \bar{\partial} \varepsilon_{\sigma_{i}} \partial \varepsilon_{\sigma_{i+1}} \cdots \partial \varepsilon_{\sigma_{n}},
\end{aligned}
$$


where $\mathfrak{S}_{n+1}$ is the permutation group of $\{0, \ldots, n\}$. Then

$$
d C_{n+1}=\pi_{n}\left(\omega_{n+1}\right), \quad \text { where } \omega_{n+1}=2^{n+1} \partial \varepsilon_{0} \wedge \cdots \wedge \partial \varepsilon_{n} \in F^{n+1}(X),
$$

and

$$
\left[C_{n+1}\left(\varepsilon_{0}, \ldots, \varepsilon_{n}\right)\right]=\left[\varepsilon_{0}\right] \cup \cdots \cup\left[\varepsilon_{n}\right] \quad \text { in } \quad H_{\mathcal{D}}^{n+1}(X, \mathbb{R}(n+1)) .
$$

Moreover for all $\sigma \in \mathfrak{S}_{n+1}$

$$
C_{n+1}\left(\varepsilon_{\sigma_{0}}, \ldots, \varepsilon_{\sigma_{n}}\right)=\operatorname{sgn} \sigma C_{n+1}\left(\varepsilon_{0}, \ldots, \varepsilon_{n}\right) .
$$

Note that for any invertible regular function $f$ on $X$ the function $\varepsilon=\log |f|$ defines an element of $H_{\mathcal{D}}^{1}(X, \mathbb{R}(1))$.

If $X$ is a variety over $\mathbb{R}$, the analytic manifold $X(\mathbb{C})$ is equipped with an antiholomorphic involution $F_{\infty}$. On the other hand we have complex conjugation on $\mathbb{C}$-valued forms $\eta$ on $X(\mathbb{C})$. We set $\bar{F}_{\infty}^{*} \eta:=F_{\infty}^{*}(\bar{\eta})=\overline{F_{\infty}^{*} \eta}$ and define the real Deligne cohomology of $X / \mathbb{R}$ by

$$
H_{\mathcal{D}}^{i}(X / \mathbb{R}, \mathbb{R}(j))=H_{\mathcal{D}}^{i}(X \otimes \mathbb{C}, \mathbb{R}(j))^{+},
$$

where + denotes the fixed space under $\bar{F}_{\infty}^{*}$. With the obvious modification-all forms have to be $\bar{F}_{\infty}^{*}$-invariant - the above theory carries over to this context.

For a nonzero Laurent polynomial $P$ in $\mathbb{C}\left[\mathbb{Z}^{n}\right]=\mathbb{C}\left[t_{1}^{ \pm 1}, \ldots, t_{n}^{ \pm 1}\right]$ set

$$
\begin{aligned}
m(P) & =\frac{1}{(2 \pi i)^{n}} \int_{T^{n}} \log \left|P\left(z_{1}, \ldots, z_{n}\right)\right| \frac{d z_{1}}{z_{1}} \wedge \cdots \wedge \frac{d z_{n}}{z_{n}} \\
& =\int_{0}^{1} \cdots \int_{0}^{1} \log \left|P\left(e^{2 \pi i \alpha_{1}}, \ldots, e^{2 \pi i \alpha_{n}}\right)\right| d \alpha_{1} \cdots d \alpha_{n} .
\end{aligned}
$$

Since $\log |P|$ is integrable over the real $n$-torus $T^{n}$, this is a real number which is known to be nonnegative if $P$ has integer coefficients [Sch], Cor. 16.6. Note that because of the formula

$$
m(P)=m\left(t_{1}^{\nu_{1}} \cdots t_{n}^{\nu_{n}} P\right) \quad \text { for } \nu_{i} \in \mathbb{Z}
$$

it would be sufficient to consider the Mahler measures of ordinary polynomials.

For $K=\mathbb{R}$ or $\mathbb{C}$ any Laurent polynomial $P$ in $K\left[\mathbb{Z}^{n}\right]$ can be viewed as a regular function on $\mathbb{G}_{m, K}^{n}$. Let $Z(P)$ be its variety of zeroes in $\mathbb{G}_{m, K}^{n}$ and write $X_{P}=$ $\mathbb{G}_{m, K}^{n} \backslash Z(P)$ for the complement. Before we can proceed we need some general notation:

For any variety $X / K$ and any subgroup $\Lambda \subset \mathbb{C}$, which in the case $K=\mathbb{R}$ should also satisfy $\bar{\Lambda}=\Lambda$, we set:

$$
H^{\nu}(X / \mathbb{C}, \Lambda)=H_{\text {sing }}^{\nu}(X(\mathbb{C}), \Lambda) \quad \text { and } \quad H^{\nu}(X / \mathbb{R}, \Lambda)=H_{\text {sing }}^{\nu}(X(\mathbb{C}), \Lambda)^{+}
$$

and similarly for homology. Set $\Lambda(k)=(2 \pi i)^{k} \Lambda \subset \mathbb{C}$. Returning to $X_{P}$ note that $F^{n+1}\left(X_{P}\right)=0$ since there are no nontrivial holomorphic $(n+1)$-forms on an $n$-dimensional variety. Hence

$$
H_{\mathcal{D}}^{n+1}\left(X_{P} / K, \mathbb{R}(n+1)\right)=H^{n}\left(X_{P} / K, \mathbb{R}(n)\right) .
$$

If $P$ is nonzero on $T^{n} \subset\left(\mathbb{C}^{*}\right)^{n}$, i.e., if $T^{n} \subset X_{P}(\mathbb{C})$, then the torus with its usual orientation defines a homology class $\left[T^{n}\right]$ in the $n$th singular homology of $X_{P}(\mathbb{C})$. If $P$ has real coefficients, so that $F_{\infty}$ acts on $X_{P}(\mathbb{C})$, we have $F_{\infty *}\left[T^{n}\right]=(-1)^{n}\left[T^{n}\right]$. For us it will be more natural to consider the class

$$
\left[T^{n}\right] \otimes(2 \pi i)^{-n} \quad \text { in } \quad H_{n}\left(X_{P} / K, \mathbb{Z}(-n)\right) .
$$


As the last ingredient we need the natural pairing:

$$
\langle,\rangle: H^{n}\left(X_{P} / K, \mathbb{R}(n)\right) \times H_{n}\left(X_{P} / K, \mathbb{R}(-n)\right) \longrightarrow \mathbb{R} .
$$

Then we have the following interpretation of $m(P)$ :

Proposition 1.2. For $K=\mathbb{R}$ or $\mathbb{C}$ consider $0 \neq P \in K\left[t_{1}^{ \pm 1}, \ldots, t_{n}^{ \pm 1}\right]$ nonzero on $T^{n} \subset\left(\mathbb{C}^{*}\right)^{n}$ and set, as above, $X_{P}=\mathbb{G}_{m, K}^{n} \backslash Z(P)$. Viewing the functions $\varepsilon_{0}=\log |P|, \varepsilon_{1}=\log \left|z_{1}\right|, \ldots, \varepsilon_{n}=\log \left|z_{n}\right|$ on $X_{P}(\mathbb{C})$ as cohomology classes $\left[\varepsilon_{i}\right]$ in $H_{\mathcal{D}}^{1}\left(X_{P} / K, \mathbb{R}(1)\right)$ we have under the isomorphism (6) that

$$
m(P)=\left\langle\left[\varepsilon_{0}\right] \cup \cdots \cup\left[\varepsilon_{n}\right],\left[T^{n}\right] \otimes(2 \pi i)^{-n}\right\rangle .
$$

Proof. The form $C_{n+1}=C_{n+1}\left(\log |P|, \log \left|z_{1}\right|, \ldots, \log \left|z_{n}\right|\right)$ of Lemma 1.1 represents $\left[\varepsilon_{0}\right] \cup \cdots \cup\left[\varepsilon_{n}\right]$ and is closed. Thus

$$
\begin{aligned}
&\left\langle\left[\varepsilon_{0}\right] \cup \cdots \cup\left[\varepsilon_{n}\right],\left[T^{n}\right] \otimes(2 \pi i)^{-n}\right\rangle=\left\langle\left[C_{n+1}\right],\left[T^{n}\right] \otimes(2 \pi i)^{-n}\right\rangle \\
&=\sum_{i=0}^{n}(-1)^{i} \frac{1}{(n+1) !} \sum_{\sigma \in \mathfrak{S}_{n}} \operatorname{sgn} \sigma \frac{1}{(2 \pi i)^{n}} \int_{T^{n}} \log |P| \frac{d \bar{z}_{\sigma_{1}}}{\bar{z}_{\sigma_{1}}} \wedge \cdots \wedge \frac{d \bar{z}_{\sigma_{i}}}{\bar{z}_{\sigma_{i}}} \\
& \wedge \frac{d z_{\sigma_{i+1}}}{z_{\sigma_{i+1}}} \wedge \cdots \wedge \frac{d z_{\sigma_{n}}}{z_{\sigma_{n}}},
\end{aligned}
$$

where $\mathfrak{S}_{n}=\left\{\sigma \in \mathfrak{S}_{n+1} \mid \sigma_{0}=0\right\}$ is the permutation group of $\{1, \ldots, n\}$. The observations needed for the last equality are that $\log \left|z_{i}\right|=0$ on $T^{n}$ and that $P$ is nonzero on $T^{n}$ by assumption. Also note that $\partial \varepsilon_{i}=\frac{1}{2} \frac{d z_{i}}{z_{i}}$ for $1 \leq i \leq n$. Using the formula

$$
\left.\frac{d \bar{z}}{\bar{z}}\right|_{S^{1}}=\frac{d z^{-1}}{z^{-1}}=-\frac{d z}{z}
$$

and anticommutativity of the wedge product, the proposition follows.

The connection of $m(P)$ with symbols in higher algebraic $K$-theory is now immediate. Namely for any regular, quasiprojective variety $X$ over a field we set, as in $[\mathrm{B} 1]$ or $[\mathrm{Schn}]$ :

$$
H_{\mathcal{M}}^{i}(X, \mathbb{Q}(j))=\operatorname{Gr}{ }_{\gamma}^{j} K_{2 j-i}(X) \otimes \mathbb{Q}
$$

the $j$ th graded piece of Quillen's algebraic $K$-group $K_{2 j-i}(X)$ with respect to the $\gamma$-filtration. It is known for example that

$$
H_{\mathcal{M}}^{1}(X, \mathbb{Q}(1))=\mathcal{O}^{*}(X) \otimes \mathbb{Q} .
$$

The product structure on $K$-theory induces a $\cup$-product on the $\mathcal{M}$-cohomologies. For varieties $X$ over $K=\mathbb{R}$ or $\mathbb{C}$ there is a natural transformation

$$
r_{\mathcal{D}}: H_{\mathcal{M}}^{i}(X, \mathbb{Q}(j)) \longrightarrow H_{\mathcal{D}}^{i}(X / K, \mathbb{R}(j))
$$

which respects the $\cup$-product structures on both sides. The map

$$
r_{\mathcal{D}}: H_{\mathcal{M}}^{1}(X, \mathbb{Q}(1)) \longrightarrow H_{\mathcal{D}}^{1}(X / K, \mathbb{R}(1))
$$

for example maps $f \in \mathcal{O}^{*}(X) \otimes \mathbb{Q}$ to $\log |f|$ viewed as a class in $H_{\mathcal{D}}^{1}(X / K, \mathbb{R}(1))$.

The iterated cup product of functions $f_{0}, \ldots, f_{n} \in \mathcal{O}^{*}(X)$ viewed as elements of $H_{\mathcal{M}}^{1}(X, \mathbb{Q}(1))$ is usually denoted by $\left\{f_{0}, \ldots, f_{n}\right\} \in H_{\mathcal{M}}^{n+1}(X, \mathbb{Q}(n+1))$ and called the symbol of $f_{0}, \ldots, f_{n}$. Thus under the assumption of 1.2 we find the following formula for the Mahler measure:

$$
m(P)=\left\langle r_{\mathcal{D}}\left\{P, t_{1}, \ldots, t_{n}\right\},\left[T^{n}\right] \otimes(2 \pi i)^{-n}\right\rangle .
$$


For a regular, quasiprojective variety $X / \mathbb{Q}$ consider the composed map:

$$
r_{\mathcal{D}}: H_{\mathcal{M}}^{i}(X, \mathbb{Q}(j)) \stackrel{\text { res }}{\longrightarrow} H_{\mathcal{M}}^{i}(X \otimes \mathbb{R}, \mathbb{Q}(j)) \stackrel{r_{\mathcal{D}}}{\longrightarrow} H_{\mathcal{D}}^{i}(X \otimes \mathbb{R}, \mathbb{R}(j)) .
$$

If $X / \mathbb{Q}$ is projective with proper regular model $\mathfrak{X} / \mathbb{Z}$, we set

$$
H_{\mathcal{M}}^{i}(X, \mathbb{Q}(j))_{\mathbb{Z}}=\operatorname{Im}\left(K_{2 j-i}(\mathfrak{X}) \otimes \mathbb{Q} \longrightarrow H_{\mathcal{M}}^{i}(X, \mathbb{Q}(j))\right) .
$$

These groups and the map $r_{\mathcal{D}}$ play an important role in the formulation of the Beilinson conjectures. If $n>\frac{j}{2}+1$ for example, the volume of $r_{\mathcal{D}}\left(H_{\mathcal{M}}^{n+1}(X, \mathbb{Q}(j))_{\mathbb{Z}}\right)$ measured against a "de Rham-Betti" $\mathbb{Q}$-structure on Deligne cohomology is expected to be given $\bmod \mathbb{Q}^{*}$ by the value at $s=j$ of the $L$-series $L\left(H^{n}(X), s\right)$; cf. [B1] or [Schn].

1.3. For $P \in \mathbb{Z}\left[t_{1}^{ \pm 1}, \ldots, t_{n}^{ \pm 1}\right]$ let $\mathfrak{X}_{P}$ denote the complement in $\mathbb{G}_{m, \mathbb{Z}}^{n}$ of its zero scheme and write $X_{P}=\mathfrak{X}_{P} \otimes \mathbb{Q}$. Then we may view the symbol $\left\{P, t_{1}, \ldots, t_{n}\right\}$ as an element of

$$
\operatorname{Im}\left(K_{n+1}\left(\mathfrak{X}_{P}\right) \otimes \mathbb{Q} \longrightarrow H_{\mathcal{M}}^{n+1}\left(X_{P}, \mathbb{Q}(n+1)\right)\right)
$$

and under the map (8), if $P$ does not vanish on $T^{n}$ we have:

$$
m(P)=\left\langle r_{\mathcal{D}}\left\{P, t_{1}, \ldots, t_{n}\right\},\left[T^{n}\right] \otimes(2 \pi i)^{-n}\right\rangle .
$$

Thus we see that $m(P)$ has an interpretation as an "integral $K$-theory cycle" evaluated against a topological cycle. It would be interesting to interpret $m(P)$ as such an evaluation on a smooth projective variety. At least in special cases this can be done; cf. section 3 .

Corollary. If $P \in \mathbb{Z}\left[t_{1}^{ \pm 1}, \ldots, t_{n}^{ \pm 1}\right]$ is nonzero on $T^{n}$ and not of the form $P=$ $c t_{1}^{a_{1}} \cdots t_{n}^{a_{n}}$, then $\left\{P, t_{1}, \ldots, t_{n}\right\} \neq 0$ in $H_{\mathcal{M}}^{n+1}\left(X_{P}, \mathbb{Q}(n+1)\right)$.

Proof. This follows from the above since $m(P) \neq 0$ for the polynomials in question; see e.g. [Sch], Th. 19.5.

\section{MAHLER MEASURE AND PERIODS OF MiXED MOtIVES IN THE EXPANSIVE CASE}

In this section we interpret $m(P)$ for a Laurent polynomial $P$ over $\mathbb{Q}$ as a Deligne period of a mixed motive. When working with the category $\mathcal{M} \mathcal{M}$ of mixed motives over $\mathbb{Q}$ we have in mind the definitions given in [De3], [Hu] or [J2]. However, any category of motives satisfying enough of the expected formal properties will do. A fairly nontechnical introduction to the notions required below can be found in [D3].

For any motive $M$ in $\mathcal{M M}$ the $G_{\mathbb{R}}=\operatorname{Gal}(\mathbb{C} / \mathbb{R})$-equivariant comparison isomorphism

$$
M_{B} \otimes \mathbb{C} \stackrel{\sim}{\longrightarrow} M_{d R} \otimes \mathbb{C}
$$

between its Betti and de Rham realizations gives rise to the perfect $\mathbb{R}$-linear period pairing

$$
\langle,\rangle_{\text {per }}:\left(M_{B} \otimes \mathbb{C}\right)^{+} \otimes_{\mathbb{R}}\left(\check{M}_{d R} \otimes \mathbb{R}\right) \longrightarrow \mathbb{R}
$$

by taking the $G_{\mathbb{R}}$-invariants. For the motive $N=\check{M}(1)$ we have $F^{0} N_{d R}=F^{1} \check{M}_{d R}$. Following Deligne and Scholl we consider the following restriction of this pairing:

$$
\langle,\rangle_{\text {per }}:\left(M_{B}^{+} \otimes \mathbb{R}\right) \otimes_{\mathbb{R}}\left(F^{0} N_{d R} \otimes \mathbb{R}\right) \longrightarrow \mathbb{R}
$$

The real numbers in the image of $M_{B}^{+} \times F^{0} N_{d R}$ under $\langle,\rangle_{\text {per }}$ are called the Deligne periods of $M$. They generate a finite-dimensional $\mathbb{Q}$-subspace of $\mathbb{R}$. In case $M$ is 
critical, i.e. (9) is nondegenerate, one can form the Deligne period determinant $c^{+}(M) \in \mathbb{R}^{*} / \mathbb{Q}^{*}$ defined as

$$
c^{+}(M)=\operatorname{det}\left(\left\langle\gamma_{i}, \omega_{j}\right\rangle_{\mathrm{per}}\right),
$$

where $\left\{\gamma_{i}\right\}$ and $\left\{\omega_{j}\right\}$ are bases of $M_{B}^{+}$and $F^{0} N_{d R}$. A motive $M$ is called integral (over $\mathbb{Z}$ ) if for all prime numbers $l \neq p$ the weight filtration on $M_{l}$ splits if $M_{l}$ is viewed as a module under the inertia group $I_{p}$ of $G_{p}=\operatorname{Gal}\left(\overline{\mathbb{Q}}_{p} / \mathbb{Q}_{p}\right)$. Let $\mathcal{M} \mathcal{M}_{\mathbb{Z}}$ be the full subcategory of $\mathcal{M M}$ of integral motives. For the definition of the $L$ series $L(M, s)$ of a motive and the relevant conjectures on its analytic behaviour we refer e.g. to [Scho1]. The following conjecture due to Deligne [De2] and Scholl [Scho1, Scho2] is the motivic version of the Beilinson conjectures:

Conjecture 2.1. For any motive $M$ in $\mathcal{M M}_{\mathbb{Z}}$, setting $N=\check{M}(1)$, we have:

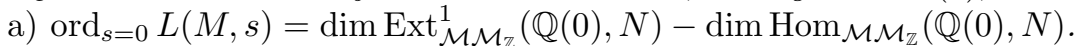

If $M$ is also critical, then:

b) $L(M, 0)=r c^{+}(M)$ for some $r \in \mathbb{Q}$.

We now proceed to Mahler measures. Let $P$ be a nonzero Laurent polynomial in $\mathbb{Q}\left[\mathbb{Z}^{n}\right]$ and define $X_{P} / \mathbb{Q}$ as the complement of its zero locus in $\mathbb{G}_{m}^{n}=\mathbb{G}_{m, \mathbb{Q}}^{n}$. We view $X_{P}$ as a closed subvariety of $\mathbb{G}_{m}^{n+1}$ via the embedding

$$
X_{P} \cong \mathbb{G}_{m}^{n+1} \cap \Gamma_{P} \hookrightarrow \mathbb{G}_{m}^{n+1},
$$

where

$$
\Gamma_{P}=\left\{\left(z_{0}, z^{\prime}\right) \in \mathbb{A}^{1} \times \mathbb{G}_{m}^{n} \mid z_{0}=P\left(z^{\prime}\right)\right\}
$$

is the graph of $P$. On $\mathbb{G}_{m}$ the group $\mu_{2}=\{ \pm 1\}$ acts by $t \mapsto t^{a}$ for $a \in \mu_{2}$. Hence we get an induced action of $\Gamma_{n+1}=\mu_{2}^{n+1} \rtimes \mathfrak{S}_{n+1}$ on $\mathbb{G}_{m}^{n+1}=\operatorname{spec} \mathbb{Q}\left[t_{0}^{ \pm 1}, \ldots, t_{n}^{ \pm 1}\right]$. Let $\varepsilon: \Gamma_{n+1} \rightarrow \mu_{2}$ be the character which is the product on $\mu_{2}^{n+1}$ and the sign character of $\mathfrak{S}_{n+1}$. In the following $\Gamma$ can be any subgroup of $\Gamma_{n+1}$ containing $\mu_{2}^{n+1}$. The restriction of $\varepsilon$ to $\Gamma$ is again denoted by $\varepsilon$. Consider

$$
X=\coprod_{\gamma \in \Gamma} X_{P}^{\gamma} \longrightarrow \mathbb{G}_{m}^{n+1}, \quad \text { resp. } \quad X=\bigcup_{\gamma \in \Gamma} X_{P}^{\gamma} \hookrightarrow \mathbb{G}_{m}^{n+1},
$$

the sum, resp. union, of the translates of $X_{P}$ under the automorphisms of $\Gamma$. With the reduced scheme structure, $X$ is a disjoint union of closed subvarieties, resp. a closed subvariety, of $\mathbb{G}_{m}^{n+1}$. In both cases $X$ is affine and $n$-dimensional. In particular we have $H^{n+1}(X)=0$ in $\mathcal{M M}$; cf. [M], VI, Th. 7.2. For any object $H$ with a $\Gamma$-action in a $\mathbb{Q}$-linear abelian category we write $H(\varepsilon)=e H$ for the $\varepsilon$-isotypical component of $H$, where $e \in \mathbb{Q}[\Gamma]$ is the idempotent corresponding to $\varepsilon$ :

$$
e=|\Gamma|^{-1} \sum_{\gamma \in \Gamma} \varepsilon(\gamma) \cdot \gamma^{-1}
$$

Then we have:

$$
H^{n}\left(\mathbb{G}_{m}^{n+1}\right)(\varepsilon)=0 \text { in } \mathcal{M M} .
$$

For this note that by the Künneth formula

$$
H^{n}\left(\mathbb{G}_{m}^{n+1}\right)=\bigoplus_{i=0}^{n} H^{1}\left(\mathbb{G}_{m}\right) \otimes \cdots \otimes H^{0}\left(\dot{\mathbb{G}}_{m}\right) \otimes \cdots \otimes H^{1}\left(\mathbb{G}_{m}\right)
$$

and that $\left(a_{0}, \ldots, a_{n}\right) \in \mu_{2}^{n+1}$ acts on the $i$ th term in the sum by multiplication with $a_{0} \cdots \hat{a}_{i} \cdots a_{n}$. 
It follows that in both cases of (10) the $\Gamma$-equivariant map $X \rightarrow \mathbb{G}_{m}^{n+1}$ gives rise to a short exact sequence in $\mathcal{M M}$ :

$$
\begin{aligned}
0 \rightarrow H^{n}(X, n+1)(\varepsilon) & \rightarrow H^{n+1}\left(\mathbb{G}_{m}^{n+1} \operatorname{rel} X, n+1\right)(\varepsilon) \\
& \stackrel{p}{\rightarrow} H^{n+1}\left(\mathbb{G}_{m}^{n+1}, n+1\right)(\varepsilon) \rightarrow 0
\end{aligned}
$$

Here we have used the notation $H^{i}(Z, n)=H^{i}(Z)(n)$ in $\mathcal{M M}$. We have a canonical isomorphism:

$$
\mathbb{Q}(0) \stackrel{\sim}{\longrightarrow} H^{n+1}\left(\mathbb{G}_{m}^{n+1}, n+1\right)(\varepsilon)
$$

since $H^{1}\left(\mathbb{G}_{m}\right)=\mathbb{Q}(-1)$. Under the induced isomorphism

$$
\mathbb{Q}=F^{0} \mathbb{Q}(0)_{d R} \stackrel{\sim}{\longrightarrow} F^{n+1} H_{d R}^{n+1}\left(\mathbb{G}_{m}^{n+1} / \mathbb{Q}\right)(\varepsilon),
$$

$1 \in \mathbb{Q}$ corresponds to the class of the invariant $(n+1)$-form $\omega=\frac{d t_{0}}{t_{0}} \wedge \cdots \wedge \frac{d t_{n}}{t_{n}}$. The motives

$$
N=H^{n+1}\left(\mathbb{G}_{m}^{n+1} \operatorname{rel} X, n+1\right)(\varepsilon)
$$

for $X=\coprod_{\gamma \in \Gamma} X_{P}^{\gamma}$, resp. $X=\bigcup_{\gamma \in \Gamma} X_{P}^{\gamma}$, therefore are extensions:

$$
0 \longrightarrow H^{n}(X, n+1)(\varepsilon) \longrightarrow N \longrightarrow \mathbb{Q}(0) \longrightarrow 0,
$$

and we denote by $\omega_{\mathcal{H}} \in F^{0} N_{d R}$ the canonical class corresponding to $1 \in \mathbb{Q}$ under the isomorphism

$$
F^{0} N_{d R} \stackrel{\sim}{\longrightarrow} F^{0} \mathbb{Q}(0)_{d R}=\mathbb{Q} .
$$

On the other hand, the twisted dual

$$
M=\check{N}(1)=H_{n+1}\left(\mathbb{G}_{m}^{n+1} \operatorname{rel} X,-n\right)(\varepsilon)
$$

is an extension

$$
0 \longrightarrow \mathbb{Q}(1) \longrightarrow M \longrightarrow H_{n}(X,-n)(\varepsilon) \longrightarrow 0
$$

so that

$$
M_{B}^{+} \cong H_{n}^{B}(X, \mathbb{Q}(-n))^{+}(\varepsilon)
$$

since $\mathbb{Q}(1)_{B}^{+}=0$. Now assume that $P$ does not vanish on $T^{n} \subset\left(\mathbb{C}^{*}\right)^{n}$, i.e. that $T^{n} \subset X_{P}(\mathbb{C})$. Then $T^{n}$ defines a homology class $\left[T^{n}\right]$ in $H_{n}\left(X_{P}(\mathbb{C}), \mathbb{Q}\right)$ and we let $i_{*}\left[T^{n}\right](\varepsilon)$ be the $\varepsilon$-isotypical component of $i_{*}\left[T^{n}\right]$ in $H_{n}(X(\mathbb{C}), \mathbb{Q})$, where $i: X_{P} \hookrightarrow$ $X$ is the inclusion. Let $c \in M_{B}^{+}$be the element corresponding to the cycle

$$
i_{*}\left[T^{n}\right](\varepsilon) \otimes(2 \pi i)^{-n} \quad \text { in } \quad H_{n}^{B}(X, \mathbb{Q}(-n))^{+}(\varepsilon) .
$$

Then we show:

Theorem 2.2. For $P \in \mathbb{Q}\left[t_{1}^{ \pm 1}, \ldots, t_{n}^{ \pm 1}\right]$ without zeroes on $T^{n}$ we have, under the period pairing

that

$$
\langle,\rangle_{\text {per }}: M_{B}^{+} \times F^{0} N_{d R} \longrightarrow \mathbb{R}
$$

$$
\left\langle c, \omega_{\mathcal{H}}\right\rangle_{\text {per }}=m(P) .
$$

If $P$ has integer coefficients, we thus get an interpretation of $\left\langle c, \omega_{\mathcal{H}}\right\rangle_{\text {per }}$ as the entropy of a natural $\mathbb{Z}^{n}$-action.

Remark 2.3. Although the action of $\mu_{2}^{n+1}$ is sufficient for the statement of the theorem, the action of a larger group can be useful. If for example $P$ is symmetric the consideration of the $\varepsilon$-isotypical components with respect to the larger group $\mu_{2}^{n+1} \ltimes \mathfrak{S}_{n}$, where $\mathfrak{S}_{n}=\left\{\sigma \in \mathfrak{S}_{n+1} \mid \sigma_{0}=0\right\}$, will cut down the motive $N$ bringing it closer to being critical. 
Proof. We first note that it is sufficient to prove the result for $X=\coprod_{\gamma} X_{P}^{\gamma}$. To see this set $X^{\prime}=\bigcup_{\gamma} X_{P}^{\gamma}$ and write $N^{\prime}=H^{n+1}\left(\mathbb{G}_{m}^{n+1}\right.$ rel $\left.X^{\prime}, n+1\right)(\varepsilon)$ and $M^{\prime}=$ $\check{N}^{\prime}(1)=H_{n+1}\left(\mathbb{G}_{m}^{n+1}\right.$ rel $\left.X^{\prime},-n\right)(\varepsilon)$ and correspondingly $\omega_{\mathcal{H}}^{\prime} \in F^{0} N_{d R}^{\prime}$ and $c^{\prime} \in$ $M_{B}^{\prime+}$ for the moment. The natural map $\alpha: X \rightarrow X^{\prime}$ induces a commutative diagram:

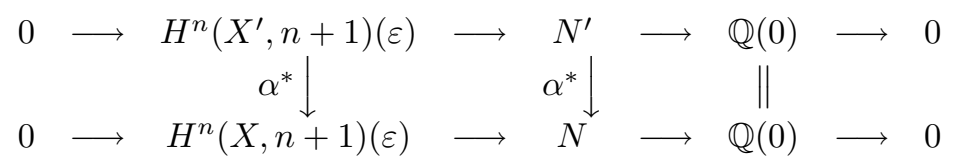

and hence commutative diagrams:

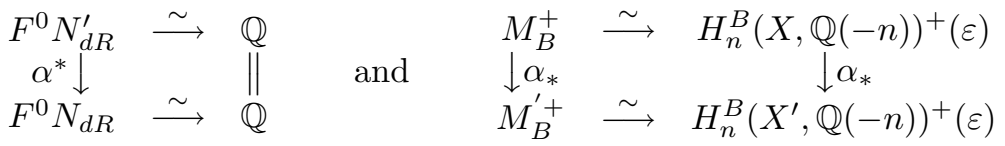

which imply that $\alpha^{*} \omega_{\mathcal{H}}^{\prime}=\omega_{\mathcal{H}}$ and $\alpha_{*} c=c^{\prime}$. Using this remark and the commutativity of the diagram of period pairings

$$
\begin{array}{cccccc}
M_{B}^{+} & \times & F^{0} N_{d R} & \stackrel{\langle,\rangle_{\text {per }}}{\longrightarrow} & \mathbb{R} \\
\alpha_{*} \downarrow & & \uparrow \alpha^{*} & & \\
M_{B}^{\prime+} & \times & F^{0} N_{d R}^{\prime} & \stackrel{\langle,\rangle_{\text {per }}}{\longrightarrow} & \mathbb{R}
\end{array}
$$

we see that

$$
\left\langle c, \omega_{\mathcal{H}}\right\rangle_{\text {per }}=\left\langle c, \alpha^{*} \omega_{\mathcal{H}}^{\prime}\right\rangle_{\text {per }}=\left\langle\alpha_{*} c, \omega_{\mathcal{H}}^{\prime}\right\rangle_{\text {per }}=\left\langle c^{\prime}, \omega_{\mathcal{H}}^{\prime}\right\rangle_{\text {per }}
$$

Hence it is sufficient to verify the equation

$$
\left\langle c, \omega_{\mathcal{H}}\right\rangle_{\mathrm{per}}=m(P)
$$

in case $X$ is the smooth variety $X=\coprod_{\gamma} X_{P}^{\gamma}$. For this we require some preparations on $C^{\infty}$-resolutions: On a smooth complex variety $V$ let $\mathcal{A}_{V}, \mathcal{A}_{\dot{V}}$ be the de Rham complex of $\mathbb{C}$-valued $C^{\infty}$-forms, resp. the Dolbeault double complex of $C^{\infty}(p, q)$ forms. By a small $s$ we denote the associated simple complex of a double complex, e.g. $\mathcal{A}_{V}=s \mathcal{A}_{\dot{V}}$. For the proof of the next result see e.g. [J1], 1.7:

Lemma. Let $V / \mathbb{C}$ be smooth and proper, and let $U \subset V$ be an open subvariety such that $Y=V \backslash U$ is a divisor with only normal crossings. Then

$$
\begin{aligned}
A_{V}^{\bullet}\langle Y\rangle & =s\left(\Omega_{V}^{\bullet}\langle Y\rangle \otimes_{\Omega_{V}^{\bullet}} \mathcal{A}_{V}^{\bullet}\right), \\
F^{i} \mathcal{A}_{V}\langle Y\rangle & =s\left(F^{i} \Omega_{V}^{\bullet}\langle Y\rangle \otimes_{\Omega_{V}^{\bullet}} \mathcal{A}_{V}^{\bullet}\right)=\bigoplus_{\substack{p \geq i \\
p+q=\bullet}} \Omega_{V}^{p}\langle Y\rangle \otimes_{\mathcal{O}_{V}} \mathcal{A}_{V}^{0, q}
\end{aligned}
$$

are complexes of fine sheaves on $V^{\text {an }}$ and the inclusion $\left(\Omega_{V}^{\bullet}\langle Y\rangle, F^{i}\right) \hookrightarrow\left(\mathcal{A}_{V}\langle Y\rangle, F^{i}\right)$ is a filtered quasi-isomorphism.

Thus we get isomorphisms

$$
H^{k}(U, \mathbb{C})=H^{k}\left(V, \Omega_{V}^{\bullet}\langle Y\rangle\right)=H^{k}\left(\Gamma\left(V, \mathcal{A}_{V}^{\bullet}\langle Y\rangle\right)\right)
$$

and

$$
\begin{aligned}
F^{i} H^{k}(U, \mathbb{C}) & :=\operatorname{Im}\left(H^{k}\left(V, F^{i} \Omega_{V}^{\bullet}\langle Y\rangle\right) \hookrightarrow H^{k}\left(V, \Omega_{V}\langle Y\rangle\right)\right) \\
& =\operatorname{Im}\left(H^{k}\left(\Gamma\left(V, F^{i} \mathcal{A}_{V}\langle Y\rangle\right)\right) \hookrightarrow H^{k}\left(\Gamma\left(V, \mathcal{A}_{V}\langle Y\rangle\right)\right)\right)
\end{aligned}
$$


For a smooth variety $\tilde{X} / \mathbb{C}$ and a map $\rho: \tilde{X} \rightarrow U \subset V$ we have

$$
\begin{aligned}
H^{k+1}(U \operatorname{rel} \tilde{X}, \mathbb{C}) & =H^{k+1}\left(\operatorname{Cone}\left(\Gamma\left(V, \mathcal{A}_{V}^{\bullet}\langle Y\rangle\right) \stackrel{\rho^{*}}{\longrightarrow} \Gamma\left(\tilde{X}, \mathcal{A}_{\tilde{X}}^{\bullet}\right)\right)[-1]\right) \\
& =H^{k}\left(\Gamma\left(V, \mathcal{A}_{V}^{++1}\langle Y\rangle\right) \oplus \Gamma\left(\tilde{X}, \mathcal{A}_{\tilde{X}}^{\bullet}\right)\right)
\end{aligned}
$$

with the differential on the direct sum given by:

$$
d(\psi, \xi)=\left(-d \psi, \rho^{*} \psi+d \xi\right) .
$$

Furthermore, it follows e.g. from the proof of [De1], Prop. 8.3.9, that

$$
F^{i} H^{k+1}(U \operatorname{rel} \tilde{X}, \mathbb{C}) \supset \operatorname{Im}\left(H^{k}\left(\Gamma\left(V, F^{i} \mathcal{A}_{V}^{\bullet+1}\langle Y\rangle\right) \oplus 0 \longrightarrow H^{k+1}(U \operatorname{rel} \tilde{X}, \mathbb{C})\right) .\right.
$$

Applying this to $U=\mathbb{G}_{m, \mathbb{C}}^{n+1}, V=\left(\mathbb{P}_{\mathbb{C}}^{1}\right)^{n+1}, Y=V \backslash U$ and the natural map $\rho: \tilde{X}=$ $X \otimes_{\mathbb{Q}} \mathbb{C} \rightarrow \mathbb{G}_{m, \mathbb{C}}^{n+1}$ we see that the exact sequence of $\mathbb{C}$-vector spaces underlying the de Rham realization over $\mathbb{C}$ of (12), (13) becomes

$$
\begin{aligned}
0 \longrightarrow H^{n}\left(\Gamma\left(\tilde{X}, \mathcal{A}^{\bullet}\right)\right)(\varepsilon) & \longrightarrow H^{n}\left(\Gamma\left(V, \mathcal{A}_{V}^{+1}\langle Y\rangle\right) \oplus \Gamma\left(\tilde{X}, \mathcal{A}_{\tilde{X}}^{\bullet}\right)\right)(\varepsilon) \\
& \stackrel{p}{\longrightarrow} H^{n}\left(\Gamma\left(V, \mathcal{A}_{V}^{++1}\langle Y\rangle\right)\right)(\varepsilon) \longrightarrow 0,
\end{aligned}
$$

where $p[(\psi, \xi)]=[\psi]$. The element

$$
(\omega, 0) \in \Gamma\left(V, F^{n+1} \mathcal{A}_{V}^{n+1}\langle Y\rangle\right) \oplus 0 \subset \Gamma\left(V, \mathcal{A}_{V}^{n+1}\langle Y\rangle\right) \oplus \Gamma\left(\tilde{X}, \mathcal{A}_{\tilde{X}}^{n}\right)
$$

is closed since $\omega$ is and since $\tilde{X}$ is $n$-dimensional. According to (15) it defines a cohomology class

$$
[(\omega, 0)] \in F^{n+1} H^{n+1}(U \operatorname{rel} \tilde{X}, \mathbb{C})(\varepsilon) .
$$

Since $p[(\omega, 0)]=[\omega]$ and since $[\omega] \in F^{n+1} H_{d R}^{n+1}\left(\mathbb{G}_{m}^{n+1} / \mathbb{Q}\right)(\varepsilon)$, it follows from the vanishing of $F^{n+1} H^{n}\left(\Gamma\left(\tilde{X}, \mathcal{A}^{\bullet}\right)\right)$ that $[(\omega, 0)]$ is defined over $\mathbb{Q}$ and hence $\omega_{\mathcal{H}}=$ $[(\omega, 0)] \in F^{0} N_{d R}$. After this description of the class $\omega_{\mathcal{H}}$ we now proceed to an explicit description of the period pairing $\langle,\rangle_{\text {per }}$. As the homology of manifolds can be calculated using singular $C^{\infty}$-chains, the isomorphism (14) can be described thus:

$$
M_{B}^{+} \cong H_{n}\left(S_{\bullet+1}(U, \mathbb{Q}(-n)) \oplus S_{\bullet}(\tilde{X}, \mathbb{Q}(-n))\right)^{+}(\varepsilon) \stackrel{q}{\sim} H_{n}(S \cdot(\tilde{X}, \mathbb{Q}(-n)))^{+}(\varepsilon) .
$$

Here $S_{\bullet}(-, \mathbb{Q}(-n))$ is the homological complex of singular $C^{\infty}$-chains with coefficients in $\mathbb{Q}(-n)=(2 \pi i)^{-n} \mathbb{Q} \subset \mathbb{C}$ and the differential on $S_{\bullet+1}(U, \mathbb{Q}(-n)) \oplus$ $S_{\bullet}(\tilde{X}, \mathbb{Q}(-n))$ is given by $\partial(a, b)=\left(-\partial a+\rho_{*} b, \partial b\right)$. Finally $q$ is the projection $q[(a, b)]=[b]$. The period pairing between a class $\Omega=[(\psi, \xi)] \in N_{d R} \otimes \mathbb{R}$ viewed as a $\bar{F}_{\infty}^{*}$-invariant class in the middle group of (16) and a class $\mathfrak{Z}=[(a, b)] \in M_{B}^{+}$ is given explicitly by:

$$
\langle\mathfrak{Z}, \Omega\rangle_{\mathrm{per}}=\int_{a} \psi+\int_{b} \xi \in \mathbb{R} .
$$

Note that:

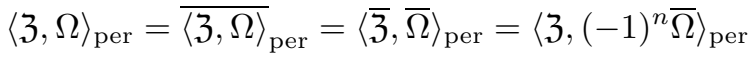

so that

$$
\langle\mathfrak{Z}, \Omega\rangle_{\mathrm{per}}=\left\langle\mathfrak{Z}, \pi_{n} \Omega\right\rangle_{\mathrm{per}} .
$$

According to Lemma 1.1 we have $\pi_{n} \omega=d C_{n+1}$, where

$$
C_{n+1}=C_{n+1}\left(\log \left|z_{0}\right|, \ldots, \log \left|z_{n}\right|\right)
$$


is the smooth $\mathbb{R}(n)$-valued $n$-form on $\mathbb{G}_{m, \mathbb{C}}^{n+1}$ defined in 1.1. Hence

$$
\pi_{n} \omega_{\mathcal{H}}=\left[\left(\pi_{n} \omega, 0\right)\right]=\left[\left(d C_{n+1}, 0\right)\right]=\left[\left(0, \rho^{*} C_{n+1}\right)\right]
$$

since

$$
\left(0, \rho^{*} C_{n+1}\right)-\left(d C_{n+1}, 0\right)=d\left(C_{n+1}, 0\right) .
$$

Finally note that $C_{n+1}$ transforms under the automorphism $\gamma \in \Gamma_{n+1}=$ $\mu_{2}^{n+1} \rtimes \mathfrak{S}_{n+1}$ according to the character $\varepsilon$ :

$$
\gamma^{*} C_{n+1}=\varepsilon(\gamma) C_{n+1}
$$

as follows from (4) for the $\mathfrak{S}_{n+1}$-operation and trivially for the $\mu_{2}^{n+1}$-operation.

As a consequence we have, for all $\gamma \in \Gamma$,

$$
\gamma^{*} \rho^{*} C_{n+1}=\varepsilon(\gamma) \rho^{*} C_{n+1}
$$

for the pullback $\rho^{*} C_{n+1}$ to $\tilde{X}=\coprod_{\gamma \in \Gamma} X_{P}^{\gamma}(\mathbb{C})$ as well. Using (18) it follows that

$$
\begin{aligned}
\left\langle c, \omega_{\mathcal{H}}\right\rangle_{\text {per }} & =\left\langle c, \pi_{n} \omega_{\mathcal{H}}\right\rangle_{\text {per }}=\left\langle c,\left[\left(0, \rho^{*} C_{n+1}\right)\right]\right\rangle_{\text {per }} \\
& =(2 \pi i)^{-n} \int_{i_{*}\left[T^{n}\right](\varepsilon)} \rho^{*} C_{n+1}=(2 \pi i)^{-n} \int_{i^{*}\left[T^{n}\right]} \rho^{*} C_{n+1},
\end{aligned}
$$

the last equality being due to (19). Thus

$$
\begin{aligned}
\left\langle c, \omega_{\mathcal{H}}\right\rangle_{\text {per }} & =(2 \pi i)^{-n} \int_{T^{n}} i^{*} \rho^{*} C_{n+1} \\
& =(2 \pi i)^{-n} \int_{T^{n}} C_{n+1}\left(\log |P|, \log \left|z_{1}\right|, \ldots, \log \left|z_{n}\right|\right)
\end{aligned}
$$

since we identified $X_{P}$ with its image in $\mathbb{G}_{m}^{n+1}$ under the graph embedding. According to Proposition (1.2) the last integral equals $m(P)$ and hence Theorem 2.2 is proved.

Remark 2.4. The relation between the motive $N$ for $X=\coprod_{\gamma \in \Gamma} X_{P}^{\gamma}$ and the symbol $\left\{P, t_{1}, \ldots, t_{n}\right\}$ is the following - at least in A. Huber's category of mixed motives defined via realizations $[\mathrm{Hu}]$. First note the canonical isomorphism:

$$
H^{n}\left(X_{P}\right) \stackrel{\sim}{\longrightarrow} H^{n}(X)(\varepsilon) \quad \text { in } \mathcal{M M} \text {. }
$$

Since $H^{n+1}\left(X_{P}\right)=0$, the construction of [Hu], Prop. 18.2.8, gives a Chern character:

$$
\begin{aligned}
\operatorname{ch}: & H_{\mathcal{M}}^{n+1}\left(X_{P}, \mathbb{Q}(n+1)\right) \\
& \rightarrow \operatorname{Ext}_{\mathcal{M M}}^{1}\left(\mathbb{Q}(0), H^{n}\left(X_{P}, n+1\right)\right)=\operatorname{Ext}_{\mathcal{M M}}^{1}\left(\mathbb{Q}(0), H^{n}(X, n+1)(\varepsilon)\right) .
\end{aligned}
$$

Using the formalism of loc. cit. as in [D2], section 5 , or [Ki], one can show that $\operatorname{ch}\left\{P, t_{1}, \ldots, t_{n}\right\}$ is represented by the extension $N$.

Remark 2.5. Related motives with $m(P)$ as a period can also be obtained as follows: Let $\mu_{2}$ act on $\mathbb{G}_{m}^{n+1}$ by involution on the first factor, and as before view $X_{P}$ as a subvariety of $\mathbb{G}_{m}^{n+1}$. Consider the natural maps

$$
X=\coprod_{\zeta \in \mu_{2}} X_{P}^{\zeta} \longrightarrow \mathbb{G}_{m}^{n+1}, \quad \text { resp. } \quad X=\bigcup_{\zeta \in \mu_{2}} X_{P}^{\zeta} \longrightarrow \mathbb{G}_{m}^{n+1},
$$

and set

$$
N=H^{n+1}\left(\mathbb{G}_{m}^{n+1} \operatorname{rel} X, n+1\right)(\delta),
$$


where $\delta$ is the nontrivial character of $\mu_{2}$. Then we have an exact sequence

$$
H^{n}(X, n+1)(\delta) \longrightarrow N \longrightarrow \mathbb{Q}(0) \longrightarrow 0,
$$

which allows us to define $\omega_{\mathcal{H}} \in F^{0} N_{d R} \cong \mathbb{Q}$ as before. The cycle $i_{*}\left[T^{n}\right](\delta) \otimes(2 \pi i)^{-n}$, where $i: X_{P} \hookrightarrow X$ is the inclusion, lies in fact in $H_{n}^{B}(X, \mathbb{Q}(-n))\left(\delta_{n+1}\right)$, where $\delta_{n+1}: \mu_{2}^{n+1} \rightarrow \mu_{2}$ is the multiplication character. Since $H_{n}^{B}\left(\mathbb{G}_{m}^{n+1}, \mathbb{Q}(-n)\right)\left(\delta_{n+1}\right)=$ 0 , we see as before that it comes from a unique class $c$ in $M_{B}^{+}$, where $M=\check{N}(1)$. The same proof as in the theorem or some commutative diagrams show that again $\left\langle c, \omega_{\mathcal{H}}\right\rangle_{\text {per }}=m(P)$.

\section{Symbols and the difference of two Mahler measures}

In this section we first derive a formula for the difference of two Mahler measures as an integral over a manifold with boundary of a representative of a Deligne cup product. We then show that in favorable cases this integral has a homological interpretation. This allows us to relate symbols with the entropy of certain nonexpansive actions not covered by section 1 . The cohomological motivation for this approach is sketched in a simple case in the appendix to this section.

In the following we will consider polynomials instead of Laurent polynomials to simplify certain formulas. In view of $(5)$ this means no loss of generality.

For $0 \neq P \in \mathbb{C}\left[t_{1}, \ldots, t_{n}\right]$,

$$
P\left(t_{1}, \ldots, t_{n}\right)=\sum_{i \geq 0} a_{i}\left(t_{1}, \ldots, t_{n-1}\right) t_{n}^{i}
$$

set

$$
P^{*}\left(t_{1}, \ldots, t_{n-1}\right)=a_{i_{0}}\left(t_{1}, \ldots, t_{n-1}\right),
$$

where $i_{0} \geq 0$ is the smallest index $i$ for which $a_{i}$ is not the zero polynomial. Set $B=\{z \in \mathbb{C}|| z \mid \leq 1\}$ and $z^{\prime}=\left(z_{1}, \ldots, z_{n-1}\right)$, and let $Z=Z(P)$ be the (reduced) zero variety of $P$ in $\left(\mathbb{C}^{*}\right)^{n}$. Then, as has been remarked by various authors, we have the following formula:

$$
m\left(P^{*}\right)-m(P)=\frac{1}{(2 \pi i)^{n-1}} \int_{\left(T^{n-1} \times \stackrel{\circ}{ }\right) \cap Z} \log \left|z_{n}\right| \frac{d z_{1}}{z_{1}} \wedge \cdots \wedge \frac{d z_{n-1}}{z_{n-1}}:=\int_{T^{n-1}} \eta
$$

with the integrable (measurable) $(n-1)$-form on $T^{n-1}$ :

$$
\eta=\frac{1}{(2 \pi i)^{n-1}} \sum_{\substack{0 \neq b \in \mathbb{B} \\ P\left(z^{\prime}, b\right)=0}} \log |b| \frac{d z_{1}}{z_{1}} \wedge \cdots \wedge \frac{d z_{n-1}}{z_{n-1}} .
$$

Here, for those $z^{\prime} \in T^{n-1}$ such that $P_{z^{\prime}}(t)=P\left(z^{\prime}, t\right)$ is not the zero polynomial, the sum runs over the zeroes $0 \neq b \in \stackrel{\circ}{B}$ of $P_{z^{\prime}}$ counted with their multiplicities.

The set of $z^{\prime} \in T^{n-1}$ with $P_{z^{\prime}}=0$ has measure zero as follows from (3.1) below applied to $P^{*}$. Thus we may leave $\eta$ undefined there.

Fact 3.1. The set of zeroes of a polynomial $0 \neq Q \in \mathbb{C}\left[t_{1}, \ldots, t_{k}\right]$ on $T^{k}$ has measure zero with respect to the Haar measure of $T^{k}$.

This follows e.g. by inductively analysing the projection to the first $k-1$ coordinates $Z(Q) \cap T^{k} \rightarrow T^{k-1}$. 
The proof of (20) uses Jensen's formula in one variable (e.g. [A], p. 206), which states that for any holomorphic function $f \neq 0$ in $B$ we have

$$
\frac{1}{2 \pi i} \int_{S^{1}} \log |f(z)| \frac{d z}{z}=\log |\tilde{f}(0)|-\sum_{\substack{0 \neq b \in \mathcal{B} \\ f(b)=0}} \log |b|,
$$

where $\tilde{f}(z)=z^{-\operatorname{ord}_{0}(f)} f(z)$ and where we sum over the zeroes $0 \neq b \in \stackrel{\circ}{B}$ of $f$ counted with their multiplicities.

It follows from this and (3.1) that for all $z^{\prime} \in T^{n-1}$ which are not in the set of measure zero $T^{n-1} \cap Z\left(P^{*}\right)$ we have:

$$
\frac{1}{2 \pi i} \int_{S^{1}} \log \left|P\left(z^{\prime}, z_{n}\right)\right| \frac{d z_{n}}{z_{n}}=\log \left|P^{*}\left(z^{\prime}\right)\right|-\sum_{\substack{0 \neq b \in \mathbb{B} \\ P\left(z^{\prime}, b\right)=0}} \log |b| .
$$

Now (20) follows immediately.

For a relation of Jensen's formula with the tame symbol on $K_{2}$ see the appendix to this section.

To proceed we impose certain conditions on the polynomial $P$.

Assumptions 3.2. 1) The divisor $\operatorname{div} P$ of $P$ in $\mathbb{G}_{m}^{n-1} \times \mathbb{A}^{1}$ over $\mathbb{C}$ has no multiple components. Moreover, $\left(T^{n-1} \times\{0\}\right) \cap \operatorname{supp}(\operatorname{div} P)=\emptyset$, i.e. $P^{*}\left(z^{\prime}\right)=P\left(z^{\prime}, 0\right)$ and $P^{*}$ does not vanish on $T^{n-1}$.

2) Let $A$ be the union of the connected components of dimension $n-1$ of $\overline{\left(T^{n-1} \times \stackrel{\circ}{B}\right) \cap Z} \stackrel{1)}{=} \overline{\left(T^{n-1} \times \stackrel{\circ}{B}\right) \cap \operatorname{supp}(\operatorname{div} P)}$. Thus $A$ is compact and we require that $A \subset Z^{\text {reg }}$ and that $A$ is a (real) submanifold of $\left(\mathbb{C}^{*}\right)^{n}$ with boundary.

The first condition in 1) is only assumed to simplify the formulas. The condition $\left(T^{n-1} \times\{0\}\right) \cap \operatorname{supp}(\operatorname{div} P)=\emptyset$ implies that the fibres of the projection $\left(T^{n-1} \times B\right) \cap Z \rightarrow T^{n-1}$ are finite, so that the form $\eta$ above is everywhere defined and $\operatorname{dim}\left(T^{n-1} \times \stackrel{\circ}{B}\right) \cap Z \leq n-1$. Note that the boundary of $A$ is contained in $T^{n} \cap Z^{\text {reg }}$. Since $A$ is a compact and canonically oriented submanifold of $Z^{\text {reg }}$, it defines a relative homology class

$$
[A] \in H_{n-1}\left(Z^{\mathrm{reg}}, \partial A ; \mathbb{Z}\right) .
$$

The form $\log \left|z_{n}\right| \frac{d z_{1}}{z_{1}} \wedge \cdots \wedge \frac{d z_{n-1}}{z_{n-1}}$ is smooth and integrable on $A$, and we have by (20):

$$
m\left(P^{*}\right)-m(P)=\frac{1}{(2 \pi i)^{n-1}} \int_{A} \log \left|z_{n}\right| \frac{d z_{1}}{z_{1}} \wedge \cdots \wedge \frac{d z_{n-1}}{z_{n-1}} .
$$

A similar computation as in the proof of Proposition 1.2 now gives:

Proposition 3.3. If $0 \neq P \in \mathbb{C}\left[t_{1}, \ldots, t_{n}\right]$ satisfies Assumptions 3.2 , we have:

$$
m\left(P^{*}\right)-m(P)=\frac{1}{(2 \pi i)^{n-1}} \int_{A} C_{n}\left(\log \left|z_{1}\right|, \ldots, \log \left|z_{n}\right|\right),
$$

where $C_{n}\left(\log \left|z_{1}\right|, \ldots, \log \left|z_{n}\right|\right)$ is the $(n-1)$-form on $\mathbb{G}_{m, \mathbb{C}}^{n}$ defined in Lemma 1.1.

Note that after restriction to $Z^{\text {reg }} \supset A$ the form $C_{n}$ is closed. 
We may view $C_{n}$ as defining a cohomology class $\left[C_{n}\right]$ in the relative cohomology $H^{n-1}\left(Z^{\text {reg }}, \partial A ; \mathbb{R}(n-1)\right)$ since the restriction of $C_{n}$ to $T^{n} \supset \partial A$ is zero. Evaluating $\left[C_{n}\right]$ against $[A] \otimes(2 \pi i)^{1-n} \in H_{n-1}\left(Z^{\text {reg }}, \partial A ; \mathbb{Z}(1-n)\right)$ then gives by 3.3 :

$$
m\left(P^{*}\right)-m(P)=\left\langle\left[C_{n}\right],[A] \otimes(2 \pi i)^{1-n}\right\rangle .
$$

In particular we get:

Theorem 3.4. For $K=\mathbb{R}$ or $\mathbb{C}$ and $0 \neq P \in K\left[t_{1}, \ldots, t_{n}\right]$ satisfying 3.2 assume that $\partial A=\emptyset$. Consider the associated homology class

$$
[A] \otimes(2 \pi i)^{1-n} \quad \text { in } \quad H_{n-1}\left(Z^{\mathrm{reg}} / K, \mathbb{Z}(1-n)\right)
$$

and the Deligne cohomology cup product

$$
\left[\varepsilon_{1}\right] \cup \cdots \cup\left[\varepsilon_{n}\right] \quad \text { in } H_{\mathcal{D}}^{n}\left(Z^{\mathrm{reg}} / K, \mathbb{R}(n)\right) \cong H^{n-1}\left(Z^{\mathrm{reg}} / K, \mathbb{R}(n-1)\right)
$$

of the functions $\varepsilon_{i}=\log \left|z_{i}\right|$ on $Z^{\mathrm{reg}}(\mathbb{C})$ viewed as classes $\left[\varepsilon_{i}\right]$ in $H_{\mathcal{D}}^{1}\left(Z^{\mathrm{reg}} / K, \mathbb{R}(1)\right)$. Then we have:

$$
m\left(P^{*}\right)-m(P)=\left\langle\left[\varepsilon_{1}\right] \cup \cdots \cup\left[\varepsilon_{n}\right],[A] \otimes(2 \pi i)^{1-n}\right\rangle .
$$

If $0 \neq P \in \mathbb{Q}\left[t_{1}, \ldots, t_{n}\right]$ satisfies the assumptions of the theorem and if $Z$ now denotes the (reduced) variety of zeroes of $P$ in $\mathbb{G}_{m, \mathbb{Q}}^{n}$, then the symbol $\left\{t_{1}, \ldots, t_{n}\right\}$ in $H_{\mathcal{M}}^{n}\left(Z^{\text {reg }}, \mathbb{Q}(n)\right)$ maps to $\left[\varepsilon_{1}\right] \cup \cdots \cup\left[\varepsilon_{n}\right]$ in $H_{\mathcal{D}}^{n}\left((Z \otimes \mathbb{R})^{\text {reg }}, \mathbb{R}(n)\right)$ under the regulator map and Theorem 3.4 implies:

$$
m\left(P^{*}\right)-m(P)=\left\langle r_{\mathcal{D}}\left\{t_{1}, \ldots, t_{n}\right\},[A] \otimes(2 \pi i)^{1-n}\right\rangle .
$$

Also note that if $P$ has integral coefficients, the symbol $\left\{t_{1}, \ldots, t_{n}\right\}$ comes from the $K$-theory of some integral model of $Z^{\text {reg }}$. Since 3.2 implies in particular that $P^{*}$ does not vanish on $T^{n-1}$, the preceding formula and subsection 1.3 applied to $P^{*}$ allow us to write $m(P)$ as the difference of two "(integral) $K$-theory cycles integrated against topological cycles".

Even if $\partial A \neq \emptyset$ we can sometimes get rid of relative cohomology as follows. Let $\mu_{2}$ act on $\mathbb{G}_{m}^{n}$ by involution on the last factor and assume that $Z$ is $\mu_{2}$-invariant and that $\mu_{2}$ acts trivially on $\partial A$, i.e. that the points of $\partial A \subset T^{n}$ have last coordinate \pm 1 . From the relative homology sequence of the pair $\left(Z^{\text {reg }}, \partial A\right)$ we get an isomorphism

$$
H_{n-1}\left(Z^{\text {reg }}, \mathbb{Q}(1-n)\right)(\delta) \stackrel{\sim}{\longrightarrow} H_{n-1}\left(Z^{\text {reg }}, \partial A ; \mathbb{Q}(1-n)\right)(\delta)
$$

since $H_{*}(\partial A ; \mathbb{Q})(\delta)=0$. Recall that $\delta$ was the nontrivial character of $\mu_{2}$. Let $\gamma \in H_{n-1}\left(Z^{\mathrm{reg}}, \mathbb{Q}(1-n)\right)(\delta)$ be the preimage of $[A](\delta) \otimes(2 \pi i)^{1-n}$. Then, because $\mu_{2}$ acts via $\delta$ on $C_{n}$, we get from (23) that

$$
m\left(P^{*}\right)-m(P)=\left\langle\left[C_{n}\right], \gamma\right\rangle
$$

where we now view $\left[C_{n}\right]$ as a class in $H^{n-1}\left(Z^{\text {reg }}, \mathbb{R}(n-1)\right)$. Thus we have proved:

Proposition 3.5. For $K=\mathbb{R}, \mathbb{C}$ assume that $0 \neq P \in K\left[t_{1}, \ldots, t_{n}\right]$ satisfies 3.2 and that the above discussion applies. Then we have

$$
m\left(P^{*}\right)-m(P)=\left\langle\left[\varepsilon_{1}\right] \cup \cdots \cup\left[\varepsilon_{n}\right], \gamma\right\rangle,
$$

where $\varepsilon_{i}=\log \left|z_{i}\right|$ and where the cup product is viewed as an element of

$$
H_{\mathcal{D}}^{n}\left(Z^{\mathrm{reg}} / K, \mathbb{R}(n)\right)(\delta)=H^{n-1}\left(Z^{\mathrm{reg}} / K, \mathbb{R}(n-1)\right)(\delta) .
$$

If $P$ has $\mathbb{Q}$-coefficients, we have

$$
m\left(P^{*}\right)-m(P)=\left\langle r_{\mathcal{D}}\left\{t_{1}, \ldots, t_{n}\right\}, \gamma\right\rangle,
$$

where $\left\{t_{1}, \ldots, t_{n}\right\}$ is viewed as an element of $H_{\mathcal{M}}^{n}\left(Z^{\text {reg }}, \mathbb{Q}(n)\right)(\delta)$. 
The following example suggested by D.W. Boyd led to the preceding proposition:

Example. Consider $P\left(t_{1}, t_{2}\right)=t_{1}^{2} t_{2}+t_{1} t_{2}^{2}+t_{1} t_{2}+t_{1}+t_{2}$. Then $P^{*}\left(t_{1}\right)=P\left(t_{1}, 0\right)=$ $t_{1}$ does not vanish on $S^{1}$ and 3.21) is satisfied. After some calculation we see that $A$ is a smooth curve in $S^{1} \times(B \backslash 0)$ connecting the points $(\zeta,-1)$ and $(\bar{\zeta},-1)$ on $T^{2}$, where $\zeta=\frac{1}{2}(1-i \sqrt{3})$. The variety $Z$ is smooth. It is $\mu_{2}$-invariant if -1 acts by $\sigma:\left(z_{1}, z_{2}\right) \mapsto\left(z_{1}, z_{2}^{-1}\right)$, and then $\partial A=\{(\zeta,-1),(\bar{\zeta},-1)\}$ is pointwise fixed by $\mu_{2}$. Thus all the assumptions of Proposition 3.5 are satisfied, and since $m\left(P^{*}\right)=0$ we get:

$$
m(P)=-\left\langle r_{\mathcal{D}}\left\{t_{1}, t_{2}\right\}, \gamma\right\rangle .
$$

The projective completion $E$ of $Z$ is a smooth curve of genus one in $\mathbb{P}^{2}$ :

$$
\left\{\left(w_{0}: w_{1}: w_{2}\right) \mid w_{1}^{2} w_{2}+w_{1} w_{2}^{2}+w_{0} w_{1} w_{2}+w_{0}^{2} w_{1}+w_{0}^{2} w_{2}=0\right\},
$$

where $t_{1}=w_{1} / w_{0}, t_{2}=w_{2} / w_{0}$ if $w_{0} \neq 0$. Taking the point $0=(1: 0: 0)$ as the origin, $E$ then becomes an elliptic curve over $\mathbb{Q}$. The complement of $Z$ in $E$ consists of the $\mathbb{Q}$-rational points $0, Q=(0: 0: 1), 2 Q=(0: 1:-1), 3 Q=(0: 1: 0)$, and one checks that $4 Q=0$. The functions $t_{1}$ and $t_{2}$ have the following divisors:

$$
\operatorname{div}\left(t_{1}\right)=\alpha=(0)+(Q)-(2 Q)-(3 Q)
$$

and

$$
\operatorname{div}\left(t_{2}\right)=\beta=(0)-(Q)-(2 Q)+(3 Q) .
$$

Setting $G=E \backslash Z=\langle Q\rangle \subset E_{4}$ we get an exact sequence:

$$
0 \longrightarrow H_{\mathcal{M}}^{2}(E, \mathbb{Q}(2)) \stackrel{i}{\longrightarrow} H_{\mathcal{M}}^{2}(Z, \mathbb{Q}(2)) \stackrel{\partial}{\longrightarrow} \mathbb{Q}[G]^{0} \otimes \mathbb{Q}^{*} \longrightarrow 0,
$$

where $\mathbb{Q}[G]^{0}$ is the $\mathbb{Q}$-vector space generated by the divisors $(\nu Q)-(0)$ for $\nu=$ $1,2,3$. Clearly any element in $H_{\mathcal{M}}^{2}(Z, \mathbb{Q}(2))$ which is invariant under translation $\tau_{Q}$ by $Q$ lies in the kernel of $\partial$. One checks that $\tau_{Q}\left(t_{1}, t_{2}\right)=\left(t_{2}, t_{1}^{-1}\right)$. Hence $\tau_{Q}^{*}\left\{t_{1}, t_{2}\right\}=\left\{t_{2}, t_{1}^{-1}\right\}=\left\{t_{1}, t_{2}\right\}$, and thus there is a uniquely determined element $\left[t_{1}, t_{2}\right]_{E} \in H_{\mathcal{M}}^{2}(E, \mathbb{Q}(2))$ with $i\left[t_{1}, t_{2}\right]_{E}=\left\{t_{1}, t_{2}\right\}$. Using the above formula for $m(P)$, a simple argument shows that:

$$
m(P)=-\left\langle r_{\mathcal{D}}\left[t_{1}, t_{2}\right]_{E}, \gamma\right\rangle,
$$

where $r_{\mathcal{D}}\left[t_{1}, t_{2}\right]_{E} \in H_{\mathcal{D}}^{2}(E / \mathbb{R}, \mathbb{R}(2))=H^{1}(E / \mathbb{R}, \mathbb{R}(1))$ and $\gamma$ is now viewed as an element of $H_{1}(E / \mathbb{R}, \mathbb{Q}(-1))$. We will calculate the right-hand side in terms of an Eisenstein-Kronecker series using a method due to Beilinson [B1], (4.2). Let $\Lambda \subset \mathbb{C}$ be the period lattice of the invariant differential of the first kind $\omega=$ $t_{2} t_{1}^{-1}\left(t_{2}^{2}-1\right)^{-1} d t_{1}$ on $E$, and set $A(\Lambda)=(2 \pi i)^{-1}(\bar{u} v-u \bar{v})$ with $(u, v)$ a $\mathbb{Z}$-basis of $\Lambda$ such that $\operatorname{Im}(v / u)>0$. Let $():, E(\mathbb{C}) \otimes \Lambda \rightarrow S^{1}$ be the Pontrjagin pairing given by $(z, \lambda)=\exp \left(A(\Lambda)^{-1}(z \bar{\lambda}-z \bar{\lambda})\right)$, and set $\Omega=\int_{\gamma} \omega \in \mathbb{R}$. Then we have:

Proposition. $m(P)=4 A(\Lambda) \Omega \sum_{\substack{\lambda \in \Lambda \\ \lambda \neq 0}} \frac{\lambda}{|\lambda|^{4}}(Q, \lambda)$.

Proof. The one-dimensional $\mathbb{R}$-vector space $H^{1}(E / \mathbb{R}, \mathbb{R}(1))$ is spanned by $[\omega]-[\bar{\omega}]$. For the coefficient $c$ in the relation $r_{\mathcal{D}}\left[t_{1}, t_{2}\right]_{E}=c([\omega]-[\varpi])$ we find, on the one hand, $2 c \Omega=-m(P)$, and on the other:

$$
c=(2 \pi i A(\Lambda))^{-1} \int_{E(\mathbb{C})} \omega \wedge r_{\mathcal{D}}\left[t_{1}, t_{2}\right]_{E} .
$$


Using [DW], Lemmas (1.10) and (3.2), we obtain from this

$$
m(P)=A(\Lambda) \Omega \sum_{\substack{\lambda \in \Lambda \\ \lambda \neq 0}} \sum_{g, h \in G} \alpha_{g} \beta_{h} \frac{\lambda}{|\lambda|^{4}}(g-h, \lambda),
$$

and the assertion follows after a short calculation.

Remark. To my knowledge this is the first expression of an Eisenstein-Kronecker series as a Mahler measure of a polynomial with integer coefficients.

We now explain how the above equation:

$$
m(P)=-\left\langle r_{\mathcal{D}}\left[t_{1}, t_{2}\right]_{E}, \gamma\right\rangle
$$

leads to a conjectural relation of $m(P)$ with an $L$-value. The elliptic curve $E$ has bad reduction only at the primes $p=3$ and $p=5$, and both times with Kodaira symbol $I 1$ (see below). The reductions of the divisors of $t_{1}$ and $t_{2}$ have support in the regular locus of the reduction of $E$ at $p=3,5$. Thus the remarks in [SchaScho], 3.5 , apply and we find that in fact $\left[t_{1}, t_{2}\right]_{E} \in H_{\mathcal{M}}^{2}(E, \mathbb{Q}(2))_{\mathbb{Z}}$. The Bloch-Beilinson conjectures would imply that the $\mathbb{Q}$-dimension of this group is one, and that if $0 \neq \eta \in H^{1}(E / \mathbb{R}, \mathbb{Q}(1))$, we had:

$$
r_{\mathcal{D}}\left[t_{1}, t_{2}\right]_{E}=f L^{\prime}(E, 0) \eta
$$

for some $f \in \mathbb{Q}$. Pairing with $\gamma$ and noting that $L(E, 2) \equiv(2 \pi)^{2} L^{\prime}(E, 0) \bmod \mathbb{Q}^{*}$, we would conclude that

$$
m(P) \equiv(2 \pi)^{-2} L(E, 2) \bmod \mathbb{Q}^{*},
$$

because we know that $m(P) \neq 0$. It was kindly pointed out to me by A. Tamagawa that the substitutions $x=\left(t_{1}+t_{2}\right)^{-1}$ and $y=-\left(t_{1}+t_{2}\right)^{-2} t_{1}\left(t_{1}+t_{2}+1\right)$ transform $E$ into the Weierstraß form $y^{2}+x y+y=x^{3}+x^{2}$ and hence that $E$ is isomorphic to the elliptic curve $15 \mathrm{~A}$ in the table on p. 82 of [BK]. The curve $E$ is isogenous to $X_{0}(15)$, and hence $L(E, s)=L(f, s)$, where $f$ is the unique normalized cusp form for $\Gamma_{0}(15)$. The relation between the Eisenstein-Kronecker series above and $L(E, 2)$ was observed numerically already in $[\mathrm{BlG}]$. Finally note that because of Beilinson's work on modular curves in [B1] the conjectural relation between $m(P)$ and $L(E, 2)$ could be proved if we could show the following using the Steinberg relations: Let $\varphi: X_{0}(15) \rightarrow E$ be an isogeny; then the symbol $\left\{\varphi^{*}\left(t_{1}\right), \varphi^{*}\left(t_{2}\right)\right\}$ lies in the subspace of $H_{\mathcal{M}}^{2}\left(Y_{0}(15), \mathbb{Q}(2)\right)$ generated by the symbols of modular units. Note that since $X_{0}(15)$ has $8 \mathbb{Q}$-rational points but only 4 cusps, the functions $\varphi^{*}\left(t_{1}\right), \varphi^{*}\left(t_{2}\right)$ are not modular units themselves, i.e. do not have their divisors supported in the cusps.

In general if one wishes to interpret the formula in Proposition 3.3 in terms of Deligne cohomology or even $K$-theory, it will be necessary to replace $\partial A$ by an algebraic variety. Possibly some complexification will do, however we will not pursue the question in this generality and simply consider the case where $\operatorname{dim} \partial A=0$, i.e. $n=2$ by our standing Assumptions 3.2.

Assume that for $0 \neq P \in \mathbb{Q}\left[t_{1}, t_{2}\right]$ the coordinates of the points in $\partial A \subset T^{2} \cap Z(\mathbb{C})$ are roots of unity and view $\partial A$ as a 0 -dimensional variety over $\mathbb{Q}$. As

$$
\begin{aligned}
H_{\mathcal{M}}^{1}\left(Z^{\mathrm{reg}}, \partial A ; \mathbb{Q}(1)\right) & =\operatorname{Ker}\left(H_{\mathcal{M}}^{1}\left(Z^{\mathrm{reg}}, \mathbb{Q}(1)\right) \stackrel{e v}{\rightarrow} H_{\mathcal{M}}^{1}(\partial A, \mathbb{Q}(1))\right) \\
& =\operatorname{Ker}\left(\mathcal{O}^{*}\left(Z^{\mathrm{reg}}\right) \otimes \mathbb{Q} \stackrel{e v}{\rightarrow} \bigoplus_{a \in \partial A} \kappa(a)^{*} \otimes \mathbb{Q}\right),
\end{aligned}
$$


where $e v$ is the evaluation homomorphism, we can regard $t_{1}, t_{2}$ as elements of $H_{\mathcal{M}}^{1}\left(Z^{\text {reg }}, \partial A ; \mathbb{Q}(1)\right)$, the point being that $\zeta \otimes 1=1$ in $\kappa(a)^{*} \otimes \mathbb{Q}$ for any root of unity $\zeta$. Under the regulator map the symbol

$$
\left\{t_{1}, t_{2}\right\} \in H_{\mathcal{M}}^{2}\left(Z^{\mathrm{reg}}, \partial A ; \mathbb{Q}(2)\right)
$$

is mapped to the class of $C_{2}\left(\varepsilon_{1}, \varepsilon_{2}\right)$ in

$$
H_{\mathcal{D}}^{2}\left(\left(Z^{\mathrm{reg}}, \partial A\right) / \mathbb{R}, \mathbb{R}(2)\right)=H^{1}\left(\left(Z^{\mathrm{reg}}, \partial A\right) / \mathbb{R}, \mathbb{R}(1)\right) .
$$

Evaluating against the relative homology class

$$
[A] \otimes(2 \pi i)^{-1} \in H_{1}\left(\left(Z^{\text {reg }}, \partial A\right) / \mathbb{R}, \mathbb{Z}(-1)\right)
$$

we obtain

Proposition 3.6. $m\left(P^{*}\right)-m(P)=\left\langle r_{\mathcal{D}}\left\{t_{1}, t_{2}\right\},[A] \otimes(2 \pi i)^{-1}\right\rangle$.

Example. The proposition applies to the polynomials $P\left(t_{1}, t_{2}\right)=\left(t_{1}+t_{2}\right)^{2} \pm k$ for $k=2,3$ for example, whose Mahler measures were calculated in [Sm] (see also [Sch], VI, 19.10):

$$
\begin{aligned}
& m\left(\left(t_{1}+t_{2}\right)^{2} \pm 3\right)=\frac{2}{3} \log 3+\frac{\sqrt{3}}{\pi} L\left(\chi_{3}, 2\right), \\
& m\left(\left(t_{1}+t_{2}\right)^{2} \pm 2\right)=\frac{1}{2} \log 2+\frac{2}{\pi} L\left(\chi_{4}, 2\right) .
\end{aligned}
$$

Here $\chi_{3}$ and $\chi_{4}$ are the nontrivial characters of $(\mathbb{Z} / 3)^{*}$ and $(\mathbb{Z} / 4)^{*}$. The variety $Z=Z^{\text {reg }}$ decomposes over $N=\mathbb{Q}(\sqrt{ \pm k})$ into two disjoint irreducible components both isomorphic to $\mathbb{G}_{m}$. The boundary points $\partial A \subset Z \cap T^{2}$ are contained in $\mu_{8} \times \mu_{8}$ for $k= \pm 2$ and $\mu_{12} \times \mu_{12}$ for $k= \pm 3$. Up to rational multiples the formulas (25) and (26) can be obtained from 3.6 using a known case of the Beilinson conjectures: Borel's theorem. We omit the details of such a geometric deduction of (25) and (26), but in short the point is this:

In the exact sequence

$$
H_{\mathcal{M}}^{1}(\partial A, \mathbb{Q}(2)) \longrightarrow H_{\mathcal{M}}^{2}\left(Z^{\mathrm{reg}}, \partial A ; \mathbb{Q}(2)\right) \longrightarrow H_{\mathcal{M}}^{2}\left(Z^{\mathrm{reg}}, \mathbb{Q}(2)\right) \longrightarrow 0
$$

the contribution to the term $\frac{\sqrt{3}}{\pi} L\left(\chi_{3}, 2\right)$, resp. $\frac{2}{\pi} L\left(\chi_{4}, 2\right)$, comes from $H_{\mathcal{M}}^{1}(\partial A, \mathbb{Q}(2))$ $\subset K_{3}(\partial A) \otimes \mathbb{Q}$. The terms $\frac{2}{3} \log 3$ and $\frac{1}{2} \log 2$ come from $m\left(P^{*}\right)=\log 3$, resp. $m\left(P^{*}\right)=\log 2$, and from the group

$$
H_{\mathcal{M}}^{2}\left(Z^{\mathrm{reg}}, \mathbb{Q}(2)\right)=H_{\mathcal{M}}^{2}\left(Z^{\mathrm{reg}} \otimes N, \mathbb{Q}(2)\right)^{G_{N / \mathbb{Q}}},
$$

taking into account that

$$
\begin{aligned}
H_{\mathcal{M}}^{2}\left(Z^{\text {reg }} \otimes N, \mathbb{Q}(2)\right) & \cong H_{\mathcal{M}}^{2}\left(\mathbb{G}_{m, N}, \mathbb{Q}(2)\right) \oplus H_{\mathcal{M}}^{2}\left(\mathbb{G}_{m, N}, \mathbb{Q}(2)\right) \\
& \cong\left(N^{*} \otimes \mathbb{Q}\right) \oplus\left(N^{*} \otimes \mathbb{Q}\right)
\end{aligned}
$$

Remark. In general the genus of a smooth compactification of $Z^{\text {reg }}$ will be nonzero and then, as in the preceding example, the contribution from the term $H_{\mathcal{M}}^{2}\left(Z^{\text {reg }}, \mathbb{Q}(2)\right)$ will be more interesting.

Another outlook on Proposition 3.6 is provided by the following extension of 3.3. For $L=\left(L_{1}, \ldots, L_{n}\right)$ with $L_{i} \geq 1$ consider the $L$-multiplication map on $\mathbb{G}_{m}^{n}$ which on points maps $\left(z_{1}, \ldots, z_{n}\right)$ to $\left(z_{1}^{L_{1}}, \ldots, z_{n}^{L_{n}}\right)$. Since clearly

$$
L^{*} C_{n}\left(\log \left|z_{1}\right|, \ldots, \log \left|z_{n}\right|\right)=L_{1} \cdots L_{n} C_{n}\left(\log \left|z_{1}\right|, \ldots, \log \left|z_{n}\right|\right),
$$


it follows from 3.3 that if $0 \neq P \in \mathbb{C}\left[t_{1}, \ldots, t_{n}\right]$ satisfies 3.2 , we have

$$
m\left(P^{*}\right)-m(P)=\frac{1}{(2 \pi i)^{n-1}}\left(L_{1} \cdots L_{n}\right)^{-1} \int_{L(A)} C_{n}\left(\log \left|z_{1}\right|, \ldots, \log \left|z_{n}\right|\right) .
$$

If for example $n=2$ and $\partial A \subset \mu_{L_{1}} \times \mu_{L_{2}}$, it follows that $L(A)$ is a union of closed curves in the usually singular variety $L\left(Z^{\text {reg }}\right) \subset \mathbb{G}_{m}^{2}$ and $(27)$ gains a homological meaning in this context. The interpretation in 3.6, while similar in spirit, has the advantage though that it deals only with nonsingular varieties.

Appendix: Jensen's formula and the tame symbol. Here we sketch how Jensen's formula (21) for polynomials is related to the tame symbol on $K_{2}$. This is probably known to the experts. Let $P \in \mathbb{C}[z]$ be a polynomial without zeroes on $S^{1}=\partial B$. According to $(7)$ we have the following formula:

$$
m(P)=\left\langle r_{\mathcal{D}}\{P, z\},\left[S^{1}\right] \otimes(2 \pi i)^{-1}\right\rangle .
$$

Let $Z$ be the (reduced) variety of zeroes of $P$ in $\mathbb{G}_{m}$ and $i: \tilde{Z}=Z \cup\{0\} \hookrightarrow \mathbb{A}^{1}$ the closed immersion (everything over $\mathbb{C}$ ). Consider the diagram

$$
\begin{aligned}
& H_{\mathcal{M}}^{2}\left(\mathbb{G}_{m} \backslash Z, \mathbb{Q}(2)\right)=H_{\mathcal{M}}^{2}\left(\mathbb{A}^{1} \backslash \tilde{Z}, \mathbb{Q}(2)\right) \stackrel{\partial}{\longrightarrow} H_{\mathcal{M}, \tilde{Z}}^{3}\left(\mathbb{A}^{1}, \mathbb{Q}(2)\right) \\
& \downarrow T \\
& \bigoplus_{b \in \tilde{Z}} \mathbb{C}^{*} \otimes \mathbb{Q} \cdot[b]=H_{\mathcal{M}}^{1}(\tilde{Z}, \mathbb{Q}(1))
\end{aligned}
$$

Here the tame symbol $T=i_{*}^{-1} \circ \partial$ is given on $\{P, Q\}$ by

$$
T\{P, Q\}=\sum_{b \in \tilde{Z}} T_{b}\{P, Q\} \cdot[b],
$$

where

$$
T_{b}\{P, Q\}=\left((-1)^{\left(\operatorname{ord}_{b} P\right)\left(\operatorname{ord}_{b} Q\right)} \frac{Q^{\operatorname{ord}_{b} P}}{P^{\operatorname{ord}_{b} Q}}\right)(b) .
$$

Hence:

$$
T_{0}\{P, z\}=(-1)^{\operatorname{ord}_{0} P} \tilde{P}(0)^{-1}, \quad \text { where } \quad \tilde{P}(z)=z^{-\operatorname{ord}_{0} P} P(z),
$$

and for $b \neq 0$ :

$$
T_{b}\{P, z\}=b^{\operatorname{ord}_{b} P} .
$$

Under the regulator map part of the diagram (A) is mapped to

$$
\begin{aligned}
H_{\mathcal{D}}^{2}\left(\mathbb{G}_{m} \backslash Z, \mathbb{R}(2)\right)=H_{\mathcal{D}}^{2}\left(\mathbb{A}^{1} \backslash \tilde{Z}, \mathbb{R}(2)\right) \stackrel{\partial}{\longrightarrow} & H_{\mathcal{D}, \tilde{Z}}^{3}\left(\mathbb{A}^{1}, \mathbb{R}(2)\right) \\
& H_{\mathcal{D}}^{1}(\tilde{Z}, \mathbb{R}(1))
\end{aligned}
$$

which in turn is isomorphic to:

$$
\begin{aligned}
& H_{B}^{1}\left(\mathbb{G}_{m} \backslash Z, \mathbb{R}(1)\right)=H_{B}^{1}\left(\mathbb{A}^{1} \backslash \tilde{Z}, \mathbb{R}(1)\right) \stackrel{\partial}{\longrightarrow} H_{B, \tilde{Z}}^{2}\left(\mathbb{A}^{1}, \mathbb{R}(1)\right) \\
& \mathbb{R}\left[\pi_{0}(\tilde{Z})\right]=H_{B}^{0}(\tilde{Z}, \mathbb{R})
\end{aligned}
$$


Thus, with $[B] \otimes(2 \pi i)^{-1} \in H_{2}^{B, \tilde{Z}}\left(\mathbb{A}^{1}, \mathbb{Z}(-1)\right)$ we find:

$$
\begin{aligned}
m(P) & =\left\langle r_{\mathcal{D}}\{P, z\}, \partial[B] \otimes(2 \pi i)^{-1}\right\rangle \\
& =\left\langle\partial r_{\mathcal{D}}\{P, z\},[B] \otimes(2 \pi i)^{-1}\right\rangle \\
& =\left\langle i_{*} r_{\mathcal{D}} T\{P, z\},[B] \otimes(2 \pi i)^{-1}\right\rangle \\
& =\sum_{b \in \tilde{Z}} \log \left|T_{b}\{P, z\}\right|\left\langle i_{*}[b],[B] \otimes(2 \pi i)^{-1}\right\rangle \\
& =-\sum_{b \in \tilde{Z} \cap B} \log \left|T_{b}\{P, z\}\right| .
\end{aligned}
$$

Hence Jensen's formula follows:

$$
m(P)=\frac{1}{2 \pi i} \int_{S^{1}} \log |P(z)| \frac{d z}{z}=\log |\tilde{P}(0)|-\sum_{\substack{0 \neq b \in B \\ P(b)=0}} \log |b|,
$$

where we count the zeroes $b$ of $P$ with their multiplicities. Under rather restrictive hypotheses on $P$ the formula of Proposition 3.3 can be established similarly.

\section{Periods of miXed motives and the Difference OF TWO MAHLER MEASURES}

In this section we give a motivic counterpart to the $K$-theoretical construction of section 3 .

For $0 \neq P \in \mathbb{Q}\left[t_{1}^{ \pm 1}, \ldots, t_{n}^{ \pm 1}\right]$ consider its (reduced) variety of zeroes $Z=Z(P)$ in $\mathbb{G}_{m}^{n} / \mathbb{Q}$. Recall the group $\Gamma_{n}=\mu_{2}^{n} \rtimes \mathfrak{S}_{n}$ and the character $\varepsilon: \Gamma_{n} \rightarrow \mu_{2}$ from section 2 . In the following, $\Gamma \subset \Gamma_{n}$ can be any subgroup containing $\mu_{2}^{n}$. Consider the $\Gamma$-equivariant maps:

$$
\hat{Z}=\coprod_{\gamma \in \Gamma} Z^{\gamma} \longrightarrow \mathbb{G}_{m}^{n}, \quad \text { resp. } \hat{Z}=\bigcup_{\gamma \in \Gamma} Z^{\gamma} \hookrightarrow \mathbb{G}_{m}^{n}
$$

Since $\hat{Z}$ is affine and $(n-1)$-dimensional, we have $H^{n}(\hat{Z})=0$. On the other hand we know by (11) that if $\varepsilon$ also denotes the restriction to $\Gamma$ of $\varepsilon: \Gamma_{n} \rightarrow \mu_{2}$, the $\varepsilon$ isotypical component with respect to the $\Gamma$-operation on $H^{n+1}\left(\mathbb{G}_{m}^{n}\right)$ vanishes. Thus the $\Gamma$-equivariant maps in $(28)$ give rise to short exact sequences:

$$
0 \longrightarrow H^{n-1}(\hat{Z}, n)(\varepsilon) \longrightarrow H^{n}\left(\mathbb{G}_{m}^{n} \operatorname{rel} \hat{Z}, n\right)(\varepsilon) \longrightarrow H^{n}\left(\mathbb{G}_{m}^{n}, n\right)(\varepsilon) \longrightarrow 0 .
$$

Set $N=H^{n}\left(\mathbb{G}_{m}^{n}\right.$ rel $\left.\hat{Z}, n\right)(\varepsilon)$. Under the canonical isomorphisms:

$$
\mathbb{Q}=F^{0} \mathbb{Q}(0)_{d R} \stackrel{\sim}{\longrightarrow} F^{n} H_{d R}^{n}\left(\mathbb{G}_{m}^{n} / \mathbb{Q}\right)(\varepsilon) \stackrel{\sim}{\longrightarrow} F^{0} N_{d R},
$$

$1 \in \mathbb{Q}$ corresponds to $\frac{d t_{1}}{t_{1}} \wedge \cdots \wedge \frac{d t_{n}}{t_{n}}$, resp. a class $\omega_{\mathcal{H}} \in F^{0} N_{d R}$. The twisted dual $M=\check{N}(1)$ is an extension:

$$
0 \longrightarrow \mathbb{Q}(1) \longrightarrow M \longrightarrow H_{n-1}(\hat{Z}, 1-n)(\varepsilon) \longrightarrow 0
$$

Thus

$$
M_{B}^{+} \cong H_{n-1}^{B}(\hat{Z}, \mathbb{Q}(1-n))^{+}(\varepsilon)
$$

Let

$$
\langle,\rangle_{\text {per }}: M_{B}^{+} \times F^{0} N_{d R} \longrightarrow \mathbb{R}
$$


be the period pairing. We can make the analogous constructions using

$$
\widehat{Z^{\mathrm{reg}}}=\coprod_{\gamma \in \Gamma}\left(Z^{\mathrm{reg}}\right)^{\gamma} \longrightarrow \mathbb{G}_{m}^{n}
$$

instead of $\hat{Z}$. The only point to notice is that we still have an exact sequence:

$$
0 \longrightarrow H^{n-1}\left(\widehat{Z^{\text {reg }}}, n\right)(\varepsilon) \longrightarrow H^{n}\left(\mathbb{G}_{m}^{n} \text { rel } \widehat{Z^{\text {reg }}}, n\right)(\varepsilon) \longrightarrow H^{n}\left(\mathbb{G}_{m}^{n}, n\right)(\varepsilon) \longrightarrow 0
$$

because $H^{n}\left(\mathbb{G}_{m}^{n}\right) \rightarrow H^{n}\left(\widehat{Z^{\text {reg }}}\right)$ is the zero map. This last fact can be seen using the $C^{\infty}$-description of the Betti realizations of the smooth manifolds $\left(\mathbb{C}^{*}\right)^{n}$ and $\widehat{Z^{\text {reg }}}(\mathbb{C})$. Since $\operatorname{dim} \widehat{Z^{\text {reg }}}=n-1$, it is clear that the generating $n$-form $\frac{d z_{1}}{z_{1}} \wedge \cdots \wedge \frac{d z_{n}}{z_{n}}$ of $H^{n}\left(\left(\mathbb{C}^{*}\right)^{n}, \mathbb{C}\right)$ is mapped to zero in $H^{n}\left(\widehat{Z^{\text {reg }}}(\mathbb{C}), \mathbb{C}\right)$. The stronger statement that there are no nontrivial maps $\mathbb{Q}(0) \rightarrow H^{n}\left(\widehat{Z^{\text {reg }}}\right)(n)$ or $\mathbb{Q}(0) \rightarrow H^{n}\left(Z^{\text {reg }}\right)(n)$, which is needed below, follows from Hodge theory because for every smooth $(n-1)$ dimensional variety $X / \mathbb{C}$ we have

$$
\operatorname{Hom}_{\mathcal{M H}}\left(\mathbb{Q}(0), H^{n}(X)(n)\right)=F^{n} H^{n}(X, \mathbb{C}) \cap H^{n}(X, \mathbb{Q}(n))=0
$$

since $F^{n} H^{n}(X, \mathbb{C})=0$. Setting $N^{\text {reg }}=H^{n}\left(\mathbb{G}_{m}^{n}\right.$ rel $\left.\widehat{Z^{\text {reg }}}, n\right)(\varepsilon)$ and $M^{\text {reg }}=$ $\check{N}^{\text {reg }}(1)$, we see that the natural maps

$$
H^{n-1}(\hat{Z}) \longrightarrow H^{n-1}\left(\widehat{Z^{\text {reg }}}\right)
$$

induce maps $N \rightarrow N^{\text {reg }}$ and $M^{\text {reg }} \rightarrow M$. A result corresponding to Theorem 3.4 is the following:

Theorem 4.1. For $0 \neq P \in \mathbb{Q}\left[t_{1}, \ldots, t_{n}\right]$ satisfying 3.2 assume $\partial A=\emptyset$. Consider the homology class:

$$
[A] \otimes(2 \pi i)^{1-n} \quad \text { in } \quad H_{n-1}^{B}\left(Z^{\mathrm{reg}}, \mathbb{Z}(1-n)\right)^{+}, \quad \text { resp. } H_{n-1}^{B}(Z, \mathbb{Z}(1-n))^{+} .
$$

Its E-isotypical component can be viewed as an element of $M_{B}^{\mathrm{reg}}{ }^{+}$, resp. of $M_{B}^{+}$, and under the period pairing we have:

$$
m\left(P^{*}\right)-m(P)=\left\langle[A](\varepsilon) \otimes(2 \pi i)^{1-n}, \omega_{\mathcal{H}}\right\rangle_{\text {per }} .
$$

Proof. By the argument in the proof of 2.2 it suffices to consider only the case

$$
\widehat{Z^{\mathrm{reg}}}=\coprod_{\gamma \in \Gamma}\left(Z^{\mathrm{reg}}\right)^{\gamma} .
$$

Since this variety is smooth over $\mathbb{Q}$, the same calculation as in loc. cit. shows that

$$
\begin{aligned}
\left\langle[A](\varepsilon) \otimes(2 \pi i)^{1-n}, \omega_{\mathcal{H}}\right\rangle_{\text {per }} & =\left.(2 \pi i)^{1-n} \int_{[A](\varepsilon)} C_{n}\left(\log \left|z_{1}\right|, \ldots, \log \left|z_{n}\right|\right)\right|_{\widehat{Z^{\text {reg }}}} \\
& =(2 \pi i)^{1-n} \int_{[A]} C_{n}\left(\log \left|z_{1}\right|, \ldots, \log \left|z_{n}\right|\right) \\
& =m\left(P^{*}\right)-m(P) \quad \text { by } 3.3 .
\end{aligned}
$$

Remarks. 1) We have canonically

$$
H^{n-1}\left(Z^{\mathrm{reg}}\right) \stackrel{\sim}{\longrightarrow} H^{n-1}\left(\widehat{Z^{\text {reg }}}\right)(\varepsilon) \quad \text { in } \mathcal{M M} .
$$


As we observed above, there are no nontrivial maps from $\mathbb{Q}(0)$ to $H^{n}\left(Z^{\text {reg }}\right)(n)$ in $\mathcal{M M}$. Thus an application of $[\mathrm{Hu}]$, Prop. 18.2.8, gives a Chern character:

$$
\begin{aligned}
\operatorname{ch}: H_{\mathcal{M}}^{n}\left(Z^{\mathrm{reg}}, \mathbb{Q}(n)\right) & \rightarrow \operatorname{Ext}_{\mathcal{M M}}^{1}\left(\mathbb{Q}(0), H^{n-1}\left(Z^{\mathrm{reg}}, n\right)\right) \\
& =\operatorname{Ext}_{\mathcal{M M}}^{1}\left(\mathbb{Q}(0), H^{n-1}\left(\widehat{Z^{\mathrm{reg}}}, n\right)(\varepsilon)\right)
\end{aligned}
$$

and one can show that $\operatorname{ch}\left\{t_{1}, \ldots, t_{n}\right\}$ is represented by $N^{\text {reg }}$.

2) Similar remarks as in 2.3, 2.5 apply in the present context as well.

Probably the theorem also holds (for $\hat{Z}$ ) if in Assumption 3.2 we drop the condition that $A$ be contained in the regular locus of $Z_{\mathbb{C}}$.

Finally we consider a motivic version of 3.6. Assume that $0 \neq P \in \mathbb{Q}\left[t_{1}, t_{2}\right]$ satisfies 3.2 and that $\partial A \subset \mu_{L_{1}} \times \mu_{L_{2}}$. For $L=\left(L_{1}, L_{2}\right)$ consider the $L$-multiplication map on $\mathbb{G}_{m}^{2}$. Let $\Gamma \subset \Gamma_{2}$ be any subgroup containing $\mu_{2}^{2}$, and consider the maps of pairs

$$
(Z, \partial A)^{\gamma}=\left(Z^{\gamma},(\partial A)^{\gamma}\right) \stackrel{L^{\gamma}}{\longrightarrow}\left(\mathbb{G}_{m}^{2}, *\right) \text { for } \gamma \in \Gamma,
$$

where $L^{\gamma}=\gamma L \gamma^{-1}$ and $*=(1,1)$. They assemble to a $\Gamma$-equivariant map

$$
\hat{L}:(Z, \partial A)^{\wedge}=\coprod_{\gamma \in \Gamma}(Z, \partial A)^{\gamma} \longrightarrow\left(\mathbb{G}_{m}^{2}, *\right) .
$$

Because of $H^{2}(Z, \partial A)=0$ we get a short exact sequence:

$$
\begin{aligned}
0 \longrightarrow H^{1}\left((Z, \partial A)^{\wedge}, 2\right)(\varepsilon) \longrightarrow H^{2}\left(\left(\mathbb{G}_{m}^{2}, *\right) \operatorname{rel}(Z, \partial A)^{\wedge}, 2\right)(\varepsilon) \\
\quad \longrightarrow H^{2}\left(\mathbb{G}_{m}^{2}, 2\right)(\varepsilon) \longrightarrow 0 .
\end{aligned}
$$

Set $N=H^{2}\left(\left(\mathbb{G}_{m}^{2}, *\right) \operatorname{rel}(Z, \partial A)^{\wedge}, 2\right)(\varepsilon)$, and let $\omega_{\mathcal{H}} \in F^{0} N_{d R}$ be as usual the class corresponding to $\frac{d t_{1}}{t_{1}} \wedge \frac{d t_{2}}{t_{2}}$ in $F^{2} H_{d R}^{2}\left(\mathbb{G}_{m}^{2} / \mathbb{Q}\right)(\varepsilon)$. For the twisted dual $M=\check{N}(1)$ we have

$$
M_{B}^{+} \cong H_{1}^{B}\left((Z, \partial A)^{\wedge}, \mathbb{Q}(-1)\right)^{+}(\varepsilon) .
$$

Since $H^{2}\left(Z^{\text {reg }}, \partial A\right)=0$, we can make the analogous construction with $Z^{\text {reg }}$ instead ot $Z$. Call $N^{\text {reg }}$ and $M^{\text {reg }}$ the corresponding motives.

Theorem 4.2. In the above situation consider the homology class

$$
[A] \otimes(2 \pi i)^{-1} \quad \text { in } H_{1}^{B}\left(Z^{\mathrm{reg}}, \partial A ; \mathbb{Z}(-1)\right), \text { resp. } H_{1}^{B}(Z, \partial A ; \mathbb{Z}(-1))
$$

and view its $\varepsilon$-isotypical component as an element of $M_{B}^{\mathrm{reg}+}$, resp. $M_{B}^{+}$. Then we have

$$
m\left(P^{*}\right)-m(P)=L_{1}^{-1} L_{2}^{-1}\left\langle[A](\varepsilon) \otimes(2 \pi i)^{-1}, \omega_{\mathcal{H}}\right\rangle_{\mathrm{per}} .
$$

Proof. It suffices to consider the case $N^{\text {reg }}$. An adaptation of the proof of 2.2 then shows that

$$
\begin{aligned}
\left\langle[A](\varepsilon) \otimes(2 \pi i)^{-1}, \omega_{\mathcal{H}}\right\rangle_{\text {per }} & =(2 \pi i)^{-1} \int_{[A](\varepsilon)} \hat{L}^{*} C_{2}\left(\log \left|z_{1}\right|, \log \left|z_{2}\right|\right) \\
& =(2 \pi i)^{-1} \int_{[A]} L^{*} C_{2}\left(\log \left|z_{1}\right|, \log \left|z_{2}\right|\right) \\
& =L_{1} L_{2}\left(m\left(P^{*}\right)-m(P)\right) \quad \text { by } 3.3 .
\end{aligned}
$$


Similar remarks as the ones after the proof of 4.1 apply here as well. Under the comparison with $K$-theory the symbol $\left\{t_{1}^{L_{1}}, t_{2}^{L_{2}}\right\}$ in $H_{\mathcal{M}}^{2}\left(Z^{\text {reg }}, \partial A ; \mathbb{Q}(2)\right)$ is mapped by the Chern character to the class of $N^{\text {reg }}$ in $\operatorname{Ext}_{\mathcal{M M}}^{1}\left(\mathbb{Q}(0), H^{1}\left(Z^{\text {reg }}, \partial A\right)(2)\right)$, noting that $H^{1}\left(Z^{\text {reg }}, \partial A\right) \cong H^{1}\left(\left(Z^{\text {reg }}, \partial A\right)^{\wedge}\right)(\varepsilon)$.

It seems probable to me that using the natural one-extension of the motive $H^{n-1}(L(Z), n)$ coming from the embedding $L(Z) \subset \mathbb{G}_{m}^{n}$ one can treat the case where $L(A)$ is a closed $(n-1)$-dimensional manifold also for $n>2$. The argument will be more involved though because $L(A)$ will not be contained in the regular locus of $L(Z)$.

\section{REFERENCES}

[A] L.V. Ahlfors, Complex analysis. McGraw-Hill, New York, 1966. MR 32:5844

[B1] A. Beilinson, Higher regulators and values of $L$-functions. J. Sov. Math. 30 (1985), 2036-2070. MR 86h:11103

[B2] A. Beilinson, Higher regulators of modular curves. Contemp. Math. 55, I (1986), 1-34. MR 88f: 11060

[BK] B.J. Birch, W. Kuyk eds. Modular functions of one variable IV, Springer LNM 476, 1975. MR 51:12708

[BlG] S. Bloch, D. Grayson, $K_{2}$ and $L$-functions of elliptic curves: Computer calculations. Contemp. Math. 55, I (1986), 79-88. MR 88f: 11061

[Bo1] D.W. Boyd, Speculations concerning the range of Mahler's measure. Canad. Math. Bull. 24 (1981), 453-469. MR 83h:12002

[Bo2] D.W. Boyd, Explicit formulas for Mahler's measure. Preprint 1996.

[De1] P. Deligne, Théorie de Hodge III. Publ. IHES 44 (1974), 5-78. MR 58:16653b

[De2] P. Deligne, Valeurs de fonctions $L$ et périodes d'intégrales. Proc. Symp. Pure Math. 33 (2), 1979, 313-346. MR 81d:12009

[De3] P. Deligne, Le groupe fondamental de la droite projective moins trois points. In: Ihara et al. (eds.) Galois groups over $\mathbb{Q}$, MSRI Publications, Springer 1989, 79-297. MR 90m:14016

[D1] C. Deninger, Higher regulators and Hecke $L$-series of imaginary quadratic fields I. Invent Math. 96 (1989), 1-69. MR 90f: 11041

[D2] C. Deninger, Extensions of motives associated to symmetric powers of elliptic curves and to Hecke characters of imaginary quadratic fields. Preprint 1995.

[D3] C. Deninger, On extensions of mixed motives. Preprint 1995.

[DW] C. Deninger, K. Wingberg, On the Beilinson conjectures for elliptic curves with complex multiplication. In: [RSS], 249-272. MR 89g:11045

$[\mathrm{Hu}] \quad$ A. Huber, Mixed motives and their realizations in derived categories. Springer LNM 1604, 1995.

[J1] U. Jannsen, Deligne homology, Hodge-D-conjecture and motives. In [RSS] 305-372. MR 89a:14002

[J2] U. Jannsen, Mixed motives and algebraic $K$-theory, Springer LNM 1400, 1990. MR 91g:14008

[JKS] U. Jannsen, S. Kleimann, J-P. Serre (eds.), Motives. Proc. Symp. Pure Math. 55 (1), (2), 1991. MR 94i:11003, MR 94i:11004

[Ki] G. Kings, Extensions of motives of modular forms. To appear in Math. Ann.

[LSW] D. Lind, K. Schmidt, T. Ward, Mahler measure and entropy for commuting automorphisms of compact groups. Invent Math. 101 (1990), 593-629. MR 92j:22013

[M] J.S. Milne, Étale cohomology, Princeton 1980. MR 81j:14002

[Ph] P. Philippon, Critères pour l'indépendence alǵebrique, Publ. IHES 64 (1986), 5-52. MR 88h:11048

[RSS] M. Rapoport, N. Schappacher, P. Schneider (eds.), Beilinson's conjecture on special values of $L$-functions, Perspectives in Math. 4, Academic Press 1988. MR 89a:14002

[R] G.A. Ray, Relations between Mahler's measure and values of $L$-series. Can. J. Math. 39 (1987), 694-732. MR 88m:11071 
[Sch] K. Schmidt, Dynamical systems of algebraic origin. Progress in Math. 128, Basel 1995. CMP 95:16

[SchaScho] N. Schappacher, A.J. Scholl, The boundary of the Eisenstein symbol, Math. Ann. 290 (1991), 303-321. MR 93c:11037a

[Schn] P. Schneider, Introduction to the Beilinson conjectures. In: [RSS] 1-35. MR 89g:11053

[Scho1] A.J. Scholl, Remarks on special values of $L$-functions. In: J. Coates, M.J. Taylor (eds.): $L$-functions and Arithmetic. London Math. Soc. LNS 153, 1991, 373-392. MR 92h: 11057

[Scho2] A.J. Scholl, Height pairings and special values of $L$-functions. In: [JKS] (1), 571-598. MR 95a:11061

[Sm] C.J. Smyth, On measures of polynomials in several variables. Bull. Austral. Math. Soc. 23 (1981), 49-63. MR 82k:10074

Mathematisches Institut, Westfälische Wilhelms-Universität, Einsteinstrasse 62, 48149 MÜnster, Germany

E-mail address: deninge@math.uni-muenster.de 